\title{
First upper limits from LIGO on gravitational wave bursts LIGO-P030011
}

B. Abbott, ${ }^{13}$ R. Abbott,${ }^{16}$ R. Adhikari, ${ }^{14}$ A. Ageev, ${ }^{21,28}$ B. Allen, ${ }^{40}$ R. Amin, ${ }^{35}$ S. B. Anderson, ${ }^{13}$ W. G. Anderson, ${ }^{30}$ M. Araya, ${ }^{13}$ H. Armandula, ${ }^{13}$ F. Asiri, ${ }^{13}$ - P. Aufmuth, ${ }^{32}$ C. Aulbert, ${ }^{1}$ S. Babak, ${ }^{7}$ R. Balasubramanian, ${ }^{7}$ S. Ballmer, ${ }^{14}$ B. C. Barish, ${ }^{13}$ D. Barker, ${ }^{15}$ C. Barker-Patton, ${ }^{15}$ M. Barnes, ${ }^{13}$ B. Barr, ${ }^{36}$ M. A. Barton, ${ }^{13}$ K. Bayer, ${ }^{14}$ R. Beausoleil, ${ }^{27, b}$

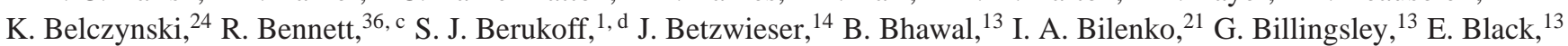
K. Blackburn, ${ }^{13}$ B. Bland-Weaver, ${ }^{15}$ B. Bochner, ${ }^{14}$, $\mathrm{L}$. Bogue, ${ }^{13}$ R. Bork,${ }^{13}$ S. Bose, ${ }^{41}$ P. R. Brady,${ }^{40}$ V. B. Braginsky, ${ }^{21}$ J. E. Brau, ${ }^{38}$ D. A. Brown, ${ }^{40}$ S. Brozek, ${ }^{32, f]}$ A. Bullington, ${ }^{27}$ A. Buonanno, ${ }^{6, g}$ R. Burgess, ${ }^{14}$ D. Busby, ${ }^{13}$ W. E. Butler, ${ }^{39}$ R. L. Byer ${ }^{27}$ L. Cadonati, ${ }^{14}$ G. Cagnoli, ${ }^{36}$ J. B. Camp, ${ }^{22}$ C. A. Cantley, ${ }^{36}$ L. Cardenas, ${ }^{13}$ K. Carter, ${ }^{16}$ M. M. Casey, ${ }^{36}$ J. Castiglione, ${ }^{35}$ A. Chandler, ${ }^{13}$ J. Chapsky, ${ }^{13, \text { h }}$ P. Charlton, ${ }^{13}$ S. Chatterji, ${ }^{14}$ Y. Chen, ${ }^{6}$ V. Chickarmane, ${ }^{17}$ D. Chin, ${ }^{37}$ N. Christensen, ${ }^{8}$ D. Churches, ${ }^{7}$ C. Colacino, ${ }^{32,2}$ R. Coldwell, ${ }^{35}$ M. Coles,,${ }^{16}$ - D. Cook, ${ }^{15}$ T. Corbitt, ${ }^{14}$ D. Coyne, ${ }^{13}$ J. D. E. Creighton, ${ }^{40}$ T. D. Creighton, ${ }^{13}$ D. R. M. Crooks, ${ }^{36}$ P. Csatorday ${ }^{14}$ B. J. Cusack, ${ }^{3}$ C. Cutler, ${ }^{1}$ E. D' Ambrosio, ${ }^{13}$ K. Danzmann, ${ }^{32,2,20}$ R. Davies, ${ }^{7}$ E. Daw, ${ }^{17},{ }^{1}$ D. DeBra, ${ }^{27}$ T. Delker, ${ }^{35}$, R. DeSalvo, ${ }^{13}$ S. Dhurandhar, ${ }^{12}$ M. Díaz, ${ }^{30}$ H. Ding, ${ }^{13}$ R. W. P. Drever, ${ }^{4}$ R. J. Dupuis,${ }^{36}$ C. Ebeling, ${ }^{8}$ J. Edlund, ${ }^{13}$ P. Ehrens, ${ }^{13}$ E. J. Elliffe,${ }^{36}$ T. Etzel, ${ }^{13}$ M. Evans, ${ }^{13}$ T. Evans, ${ }^{16}$ C. Fallnich, ${ }^{32}$ D. Farnham, ${ }^{13}$ M. M. Fejer, ${ }^{27}$ M. Fine, ${ }^{13}$ L. S. Finn, ${ }^{29}$ É. Flanagan, ${ }^{9}$ A. Freise,, 1] R. Frey, ${ }^{38}$ P. Fritschel, ${ }^{14}$ V. Frolov, ${ }^{16}$ M. Fyffe, ${ }^{16}$ K. S. Ganezer, ${ }^{5}$ J. A. Giaime, ${ }^{17}$ A. Gillespie, ${ }^{13}, \mathrm{~m}$ K. Goda, ${ }^{14}$ G. González, ${ }^{17}$ S. Goßler, ${ }^{32}$ P. Grandclément, ${ }^{24}$ A. Grant,${ }^{36}$ C. Gray, ${ }^{15}$ A. M. Gretarsson, ${ }^{16}$ D. Grimmett, ${ }^{13}$ H. Grote, ${ }^{2}$ S. Grunewald, ${ }^{1}$ M. Guenther, ${ }^{15}$ E. Gustafson, ${ }^{27}$. R. Gustafson, ${ }^{37}$ W. O. Hamilton, ${ }^{17}$ M. Hammond, ${ }^{16}$ J. Hanson, ${ }^{16}$ C. Hardham, ${ }^{27}$ G. Harry, ${ }^{14}$

A. Hartunian, ${ }^{13}$ J. Heefner, ${ }^{13}$ Y. Hefetz, ${ }^{14}$ G. Heinzel, ${ }^{2}$ I. S. Heng, ${ }^{32}$ M. Hennessy, ${ }^{27}$ N. Hepler, ${ }^{29}$ A. Heptonstall, ${ }^{36}$ M. Heurs, ${ }^{32}$ M. Hewitson, ${ }^{36}$ N. Hindman, ${ }^{15}$ P. Hoang, ${ }^{13}$ J. Hough, ${ }^{36}$ M. Hrynevych, ${ }^{13,0}$ W. Hua, ${ }^{27}$ R. Ingley, ${ }^{34}$ M. Ito, ${ }^{38}$

Y. Itoh, ${ }^{1}$ A. Ivanov, ${ }^{13}$ O. Jennrich, ${ }^{36, p}$ W. W. Johnson, ${ }^{17}$ W. Johnston, ${ }^{30}$ L. Jones, ${ }^{13}$ D. Jungwirth, ${ }^{13, q}$ V. Kalogera, ${ }^{24}$ E. Katsavounidis, ${ }^{14}$ K. Kawabe, ${ }^{20,2}$ S. Kawamura, ${ }^{23}$ W. Kells, ${ }^{13}$ J. Kern, ${ }^{16}$ A. Khan, ${ }^{16}$ S. Killbourn, ${ }^{36}$ C. J. Killow, ${ }^{36}$ C. Kim, ${ }^{24}$ C. King, ${ }^{13}$ P. King, ${ }^{13}$ S. Klimenko, ${ }^{35}$ P. Kloevekorn, ${ }^{2}$ S. Koranda, ${ }^{40}$ K. Kötter, ${ }^{32}$ J. Kovalik, ${ }^{16}$ D. Kozak, ${ }^{13}$ B. Krishnan, ${ }^{1}$ M. Landry, ${ }^{15}$ J. Langdale, ${ }^{16}$ B. Lantz, ${ }^{27}$ R. Lawrence, ${ }^{14}$ A. Lazzarini, ${ }^{13}$ M. Lei, ${ }^{13}$ V. Leonhardt,,${ }^{32}$ I. Leonor, ${ }^{38}$ K. Libbrecht, ${ }^{13}$

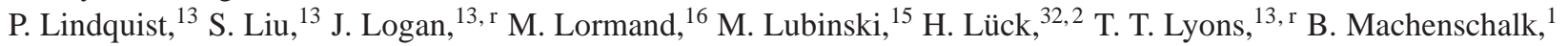
M. MacInnis, ${ }^{14}$ M. Mageswaran, ${ }^{13}$ K. Mailand, ${ }^{13}$ W. Majid,,${ }^{13, h}$ M. Malec, ${ }^{32}$ F. Mann, ${ }^{13}$ A. Marin,,${ }^{14}$ S S. Márka, ${ }^{13}$ E. Maros, ${ }^{13}$ J. Mason, ${ }^{13,6}$ K. Mason, ${ }^{14}$ O. Matherny, ${ }^{15}$ L. Matone, ${ }^{15}$ N. Mavalvala, ${ }^{14}$ R. McCarthy, ${ }^{15}$ D. E. McClelland,${ }^{3}$

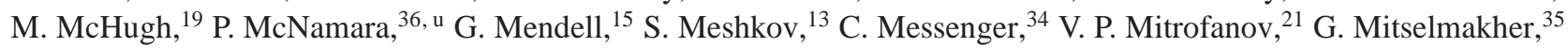
R. Mittleman, ${ }^{14}$ O. Miyakawa, ${ }^{13}$ S. Miyoki, ${ }^{13}$, V. Mohanty, ${ }^{1,}$ w G. Moreno, ${ }^{15}$ K. Mossavi, ${ }^{2}$ B. Mours, ${ }^{13, \text { }}$ G. Mueller, ${ }^{35}$

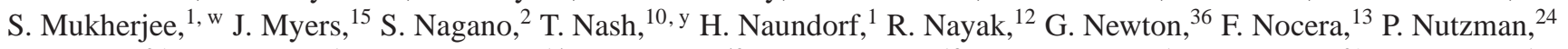

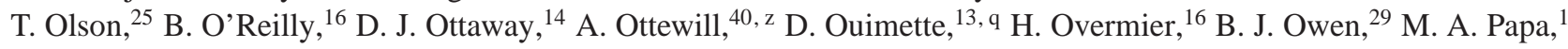
C. Parameswariah, ${ }^{16}$ V. Parameswariah, ${ }^{15}$ M. Pedraza, ${ }^{13}$ S. Penn, ${ }^{11}$ M. Pitkin, ${ }^{36}$ M. Plissi,${ }^{36}$ M. Pratt, ${ }^{14}$ V. Quetschke, ${ }^{32}$

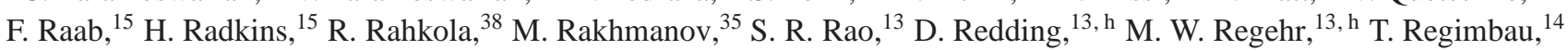
K. T. Reilly, ${ }^{13}$ K. Reithmaier, ${ }^{13}$ D. H. Reitze, ${ }^{35}$ S. Richman, ${ }^{14}$, aa R. Riesen, ${ }^{16}$ K. Riles, ${ }^{37}$ A. Rizzi, ${ }^{16}$, bb D. I. Robertson, ${ }^{36}$ N. A. Robertson, ${ }^{36,27}$ L. Robison, ${ }^{13}$ S. Roddy, ${ }^{16}$ J. Rollins, ${ }^{14}$ J. D. Romano, ${ }^{30, c c}$ J. Romie, ${ }^{13}$ H. Rong, ${ }^{35, m} \mathrm{D}$. Rose, ${ }^{13}$

E. Rotthoff, ${ }^{29}$ S. Rowan, ${ }^{36}$ A. Rüdiger, ${ }^{20,2}$ P. Russell, ${ }^{13}$ K. Ryan, ${ }^{15}$ I. Salzman, ${ }^{13}$ G. H. Sanders, ${ }^{13}$ V. Sannibale, ${ }^{13}$ B. Sathyaprakash, ${ }^{7}$ P. R. Saulson, ${ }^{28}$ R. Savage, ${ }^{15}$ A. Sazonov, ${ }^{35}$ R. Schilling, ${ }^{20,2}$ K. Schlaufman, ${ }^{29}$ V. Schmidt, ${ }^{13 \text {,dd }}$ R. Schofield, ${ }^{38}$ M. Schrempel, ${ }^{32, \text { ee }}$ B. F. Schutz, ${ }^{1,7}$ P. Schwinberg, ${ }^{15}$ S. M. Scott, ${ }^{3}$ A. C. Searle,${ }^{3}$ B. Sears, ${ }^{13}$ S. Seel, ${ }^{13}$

A. S. Sengupta, ${ }^{12}$ C. A. Shapiro, ${ }^{29, f f}$ P. Shawhan, ${ }^{13}$ D. H. Shoemaker, ${ }^{14}$ Q. Z. Shu, ${ }^{35, \text { gg }}$ A. Sibley, ${ }^{16}$ X. Siemens, ${ }^{40}$ L. Sievers, ${ }^{13, h}$ D. Sigg, ${ }^{15}$ A. M. Sintes,,${ }^{1,33}$ K. Skeldon, ${ }^{36}$ J. R. Smith, ${ }^{2}$ M. Smith, ${ }^{14}$ M. R. Smith, ${ }^{13}$ P. Sneddon, ${ }^{36}$ R. Spero, ${ }^{13, h}$

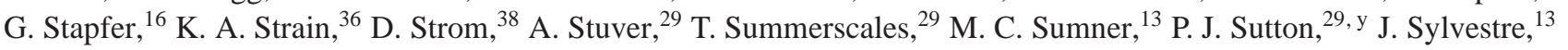

A. Takamori, ${ }^{13}$ D. B. Tanner, ${ }^{35}$ H. Tariq, ${ }^{13}$ I. Taylor, ${ }^{7}$ R. Taylor, ${ }^{13}$ K. S. Thorne, ${ }^{6}$ M. Tibbits,${ }^{29}$ S. Tilav, ${ }^{13}$,h M. Tinto,, h

K. V. Tokmakov, ${ }^{21}$ C. Torres, ${ }^{30}$ C. Torrie, ${ }^{13,36}$ S. Traeger, ${ }^{32, \text { ii }}$ G. Traylor, ${ }^{16}$ W. Tyler, ${ }^{13}$ D. Ugolini, ${ }^{31}$ M. Vallisneri, ${ }^{6, \text { ij }}$ M. van Putten, ${ }^{14}$ S. Vass, ${ }^{13}$ A. Vecchio, ${ }^{34}$ C. Vorvick, ${ }^{15}$ S. P. Vyachanin, ${ }^{21}$ L. Wallace, ${ }^{13}$ H. Walther, ${ }^{20}$ H. Ward, ${ }^{36}$ B. Ware, ${ }^{13, h}$ K. Watts, ${ }^{16}$ D. Webber, ${ }^{13}$ A. Weidner, ${ }^{20,2}$ U. Weiland, ${ }^{32}$ A. Weinstein, ${ }^{13}$ R. Weiss, ${ }^{14}$ H. Welling, ${ }^{32}$ L. Wen, ${ }^{13}$ S. Wen, ${ }^{17}$ J. T. Whelan, ${ }^{19}$ S. E. Whitcomb, ${ }^{13}$ B. F. Whiting, ${ }^{35}$ P. A. Willems, ${ }^{13}$ P. R. Williams,, kk R. Williams, ${ }^{4}$ B. Willke, ${ }^{32,2}$ A. Wilson, ${ }^{13}$ B. J. Winjum, ${ }^{29, \text { d }}$ W. Winkler,${ }^{20,2}$ S. Wise, ${ }^{35}$ A. G. Wiseman, ${ }^{40}$ G. Woan, ${ }^{36}$ R. Wooley, ${ }^{16}$ J. Worden, ${ }^{15}$ I. Yakushin, ${ }^{16}$ H. Yamamoto, ${ }^{13}$ S. Yoshida, ${ }^{26}$ I. Zawischa, ${ }^{32,11}$ L. Zhang, ${ }^{13}$ N. Zotov, ${ }^{18}$ M. Zucker, ${ }^{16}$ and J. Zweizig ${ }^{13}$

(The LIGO Scientific Collaboration, http://www.ligo.org)

${ }^{1}$ Albert-Einstein-Institut, Max-Planck-Institut für Gravitationsphysik, D-14476 Golm, Germany

${ }^{2}$ Albert-Einstein-Institut, Max-Planck-Institut für Gravitationsphysik, D-30167 Hannover, Germany

${ }^{3}$ Australian National University, Canberra, 0200, Australia

${ }^{4}$ California Institute of Technology, Pasadena, CA 91125, USA 


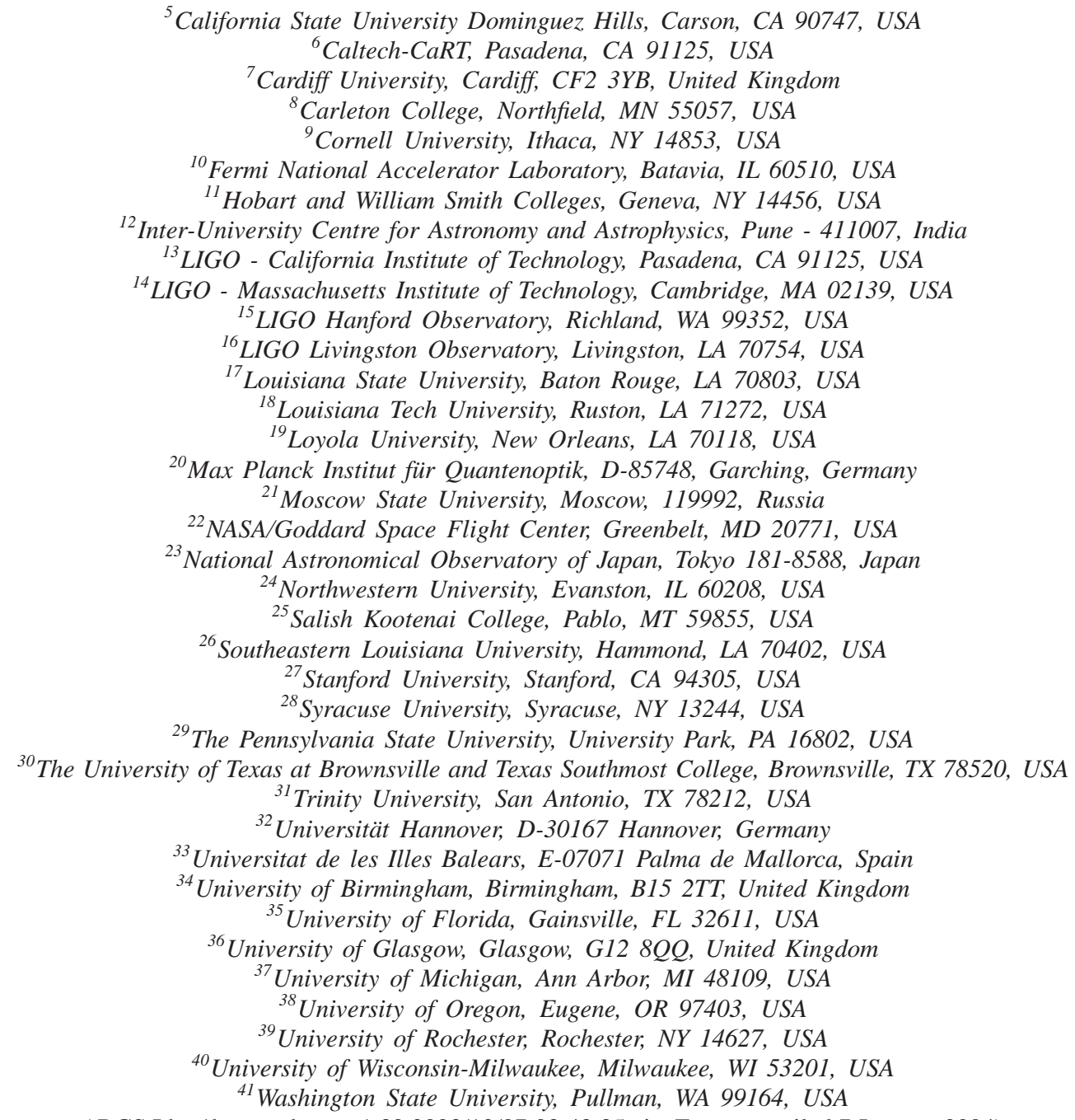

( RCS Id: s1burstprd.tex,v 1.32 2003/12/07 03:43:35 ajw Exp ; compiled 7 January 2004)

We report on a search for gravitational wave bursts using data from the first science run of the LIGO detectors. Our search focuses on bursts with durations ranging from $4 \mathrm{~ms}$ to $100 \mathrm{~ms}$, and with significant power in the LIGO sensitivity band of 150 to $3000 \mathrm{~Hz}$. We bound the rate for such detected bursts at less than 1.6 events per day at $90 \%$ confidence level. This result is interpreted in terms of the detection efficiency for ad hoc waveforms (Gaussians and sine-Gaussians) as a function of their root-sum-square strain $h_{r s s}$; typical sensitivities lie in the range $h_{r s s} \sim 10^{-19}-10^{-17}$ strain $/ \sqrt{\mathrm{Hz}}$, depending on waveform. We discuss improvements in the search method that will be applied to future science data from LIGO and other gravitational wave detectors.

PACS numbers: $04.80 . \mathrm{Nn}, 07.05 . \mathrm{Kf}, 95.30 . \mathrm{Sf}, 95.85 . \mathrm{Sz}$

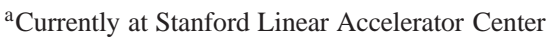

${ }^{b}$ Permanent Address: HP Laboratories

${ }^{\mathrm{c} C u r r e n t l y ~ a t ~ R u t h e r f o r d ~ A p p l e t o n ~ L a b o r a t o r y ~}$

${ }^{\mathrm{d}}$ Currently at University of California, Los Angeles

${ }^{\mathrm{e}}$ Currently at Hofstra University

${ }^{\mathrm{f}}$ Currently at Siemens AG

gPermanent Address: GReCO, Institut d'Astrophysique de Paris (CNRS)

${ }^{\text {h}}$ Currently at NASA Jet Propulsion Laboratory

${ }^{\mathrm{i}}$ Currently at National Science Foundation

${ }^{\mathrm{j} C u r r e n t l y}$ at University of Sheffield
}

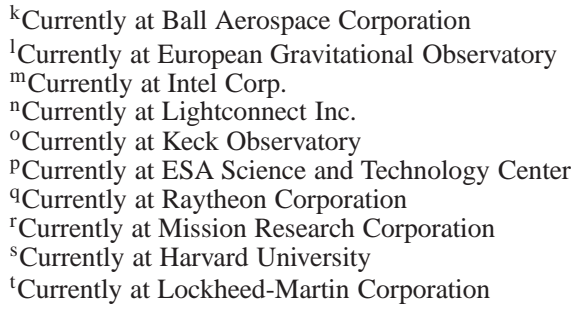




\section{INTRODUCTION}

Gravitational wave bursts are expected to be produced from astrophysical sources such as stellar collapses, the inspirals and mergers of compact binary star systems, the generators of gamma ray bursts, and other energetic phenomena. Upper limits from searches for gravitational wave bursts with resonant bar detectors have recently been reported in [1, 2, 3, 4], and results using interferometric detectors are published in [5, 6]. A new generation of detectors based on laser interferometry has been constructed, aiming for direct detection with broadband sensitivity. These include the three LIGO detectors [7] described briefly in section [I] as well as the British-German GEO 600 detector [8, 9], the Japanese TAMA 300 detector [10], and the French-Italian VIRGO detector [11], forming a worldwide network. In the summer of 2002, all three LIGO detectors were brought to their design optical configuration. After a series of engineering runs, the LIGO, GEO 600 and TAMA 300 detectors operated in coincident observation mode for the first time (Science Run 1, or S1) for two weeks in August-September 2002.

Although the LIGO detectors were far from their design sensitivity, the quality of the data was sufficiently high to exercise the first generation of analysis procedures for various types of gravitational wave searches, including searches for chirp gravitational waves from compact neutron-star binary inspirals [12], quasi-monochromatic gravitational waves from pulsar J1939+2134 [13], and broad-band stochastic background gravitational radiation [14]. In all these analyses, a well-defined astrophysical model was assumed. In this paper we report on a search (using the LIGO detectors only) for unmodeled gravitational wave bursts that might originate from supernovae in our galaxy, mergers of binary stellar-mass systems, gamma ray burst engines, or other energetic sources. The waveforms of gravitational waves from such sources are poorly known, so we employ data analysis algorithms which can, in principle, identify bursts with a broad range of possible waveforms.

The first detection of gravitational wave bursts requires sta-

\footnotetext{
uCurrently at NASA Goddard Space Flight Center

v Permanent Address: University of Tokyo, Institute for Cosmic Ray Research

${ }^{\mathrm{w}}$ Currently at The University of Texas at Brownsville and Texas Southmost College

${ }^{\mathrm{x}}$ Currently at Laboratoire d'Annecy-le-Vieux de Physique des Particules

y Currently at LIGO - California Institute of Technology

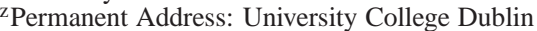

${ }^{\text {aa }}$ Currently at Research Electro-Optics Inc.

${ }^{\mathrm{bb}}$ Currently at Institute of Advanced Physics, Baton Rouge, LA

${ }^{\mathrm{cc}}$ Currently at Cardiff University

${ }^{\mathrm{dd}}$ Currently at European Commission, DG Research, Brussels, Belgium

${ }^{e e}$ Currently at Spectra Physics Corporation

${ }^{\mathrm{ff}}$ Currently at University of Chicago

gg Currently at LightBit Corporation

${ }^{\text {hh }}$ Currently at University of Delaware

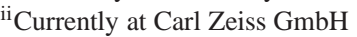

jj Permanent Address: NASA Jet Propulsion Laboratory

${ }^{\mathrm{k}}$ Currently at Shanghai Astronomical Observatory

${ }^{\text {ll } C u r r e n t l y ~ a t ~ L a s e r ~ Z e n t r u m ~ H a n n o v e r ~}$
}

ble, well understood detectors; well-tested and robust data processing procedures; and clearly defined criteria for establishing confidence that no signal is of terrestrial origin. None of these elements were firmly in place as we began this first LIGO science run; rather, this run provided the opportunity for us to understand our detectors better, exercise and hone our data processing procedures, and build confidence in our ability to establish detection of gravitational wave bursts in future science runs. Therefore, the goal for this analysis is to produce an upper limit on the rate for gravitational wave bursts, even if a purely statistical procedure suggests the presence of a signal above background. It should also be noted that the sensitivities of the three LIGO detectors during S1 were several orders of magnitude worse than required for plausible detection of bursts from astrophysical sources such as supernovae in our Milky Way galaxy [15].

In this search we focus on short (4 ms to $100 \mathrm{~ms}$ ) bursts in the LIGO sensitivity band (roughly 150 to $3000 \mathrm{~Hz}$ ), with sufficiently high strain amplitude to be observed over the detector noise. We make no other assumptions about the nature or origin of the burst. We apply software algorithms to the LIGO detector data stream to detect such bursts. In order to suppress false signals from fluctuations of the detector noise we require temporal coincidence of detected burst events in all three LIGO detectors. We estimate the rate of accidental coincidences by studying the number of time-shifted coincident burst events, and look for a statistically significant excess of coincident burst events at zero time shift. In light of the discussion in the previous paragraph, our goal for the search presented here is to set an upper limit on the rate of excess coincident bursts, given the detectors' level of sensitivity during the $\mathrm{S} 1$ run.

In order to interpret our upper limit on the rate of burst events, we evaluate the efficiency of our search algorithms for the detection of simulated bursts injected into the data streams, using simple, well-defined waveforms (Gaussians and sine-Gaussians). We obtain curves of triple-coincidence detection efficiency as a function of gravitational waveform peak amplitude at the Earth, averaged over source direction and incident wave (linear) polarization. We then combine our gravitational wave burst rate limits with these efficiency curves, yielding rate-versus-strength regions that (for the waveforms that we have examined) are excluded at the $90 \%$ confidence level or higher.

The paper is organized as follows. In section $\prod$ we briefly describe the LIGO detector array and the data obtained from the first science run, with emphasis on those characteristics most relevant for a search for short gravitational wave bursts. In section $1 \mathrm{~B}$ we briefly describe the $\mathrm{S} 1 \mathrm{run}$. In section $\llbracket \mathrm{C}$ we describe the data quality requirements that were applied to the S1 data sample, and present the subset of the data used for this search. In section $\amalg$ we describe our data processing pipeline, including the event trigger generation, event vetoes, and the time coincidence requirement. We present the results of two independent pipelines, based on the burst detection algorithms discussed in section III In section IV we estimate the background (accidental coincidence) event rate. In section $\nabla$ we evaluate the efficiency for the detection of bursts mod- 
eled with simple ad hoc waveforms, and compare that with expectations. In section VI we present our limit on the observed excess event rate. We combine this with our efficiency curves as a function of signal strength, excluding regions in the rate versus signal strength plane. We also discuss the most significant systematic errors in these measurements. We summarize these results in section VII Finally, we outline our plans to improve and expand our search methodology using data from subsequent observation runs.

\section{DETECTORS AND DATA SET}

\section{A. The LIGO detectors}

All three LIGO detectors are orthogonal arm Michelson laser interferometers. The LIGO Hanford Observatory operates two identically oriented interferometric detectors which share a common vacuum envelope: one having $4 \mathrm{~km}$ long measurement arms (referred to as $\mathrm{H} 1$ ), and one having $2 \mathrm{~km}$ long arms (H2). The LIGO Livingston Observatory operates a single $4 \mathrm{~km}$ long detector (L1). The two observatories are approximately $3000 \mathrm{~km}$ apart, corresponding to $10 \mathrm{~ms}$ of light travel time. The detectors are approximately co-aligned, so that a gravitational wave should appear with comparable signals at both sites. The principles underlying these laser interferometer gravitational wave detectors are discussed in [16]. A more detailed description of the LIGO detectors can be found in [17].

These detectors aim to detect gravitational waves by interferometrically monitoring the relative separation of mirrors which play the role of test masses, responding to space-time distortions induced by the waves as they traverse the detectors. The effect of a quadrupolar gravitational wave is to produce a strain in space, impinging upon the detector and thus displacing the mirrors at the ends of the arms by an amount proportional to the arm length. For gravitational waves incident from directly overhead or below, and polarized along the arms of the detector, the mirrors at the ends of the two arms experience purely differential motion. Waves incident from non-optimal directions and/or polarizations can also induce differential motion; the "antenna pattern" is discussed in section $\mathrm{VC}$

Each interferometer is illuminated with light from a $\mathrm{Nd}$ :YAG laser, operating at $1064 \mathrm{~nm}$ [18]. Before the light is launched into the interferometer, its frequency, amplitude and direction are all stabilized, using a combination of active and passive stabilization techniques [18, 19]. The light is sent through a beam splitter towards both arms. In each arm, a pair of mirrors (the "input test mass" and "end test mass"), separated by $2 \mathrm{~km}$ or $4 \mathrm{~km}$, form a Fabry Perot resonant optical cavity with a finesse of approximately 220. Because the Michelson interferometer antisymmetric port is held at a dark fringe, and because the Fabry-Perot cavities are low-loss, most of the light returning from the arms to the beam splitter nominally exits through the symmetric port of the beam splitter back towards the laser. A "power recycling" mirror returns it, resonantly, to the interferometer (forming a "power recycling cavity"). The average length of the arm cavities is used as a frequency reference for the final stage of frequency stabilization [17]. Differential arm cavity length changes result in a small amount of light exiting the asymmetric port of the beam splitter; this constitutes the gravitational wave signal. The effect of the arm cavities and power recycling is to increase the sensitivity of the interferometer to gravitational wave signals. The arm lengths and arm cavity finesse are optimized to minimize various noise sources.

The mirrors of the interferometer [20, 21] are suspended as pendulums [22]. Active and passive vibration isolation systems [23] are used to isolate them from seismic noise. Various feedback control systems are used to keep the multiple optical cavities tightly on resonance [24] and well aligned [25], and to keep the Michelson interferometer on a dark fringe. The L1 detector also employed feedforward control to compensate for microseismic disturbances [26]. When all length degrees of freedom are under control and the control systems are operating within their linear regime, the interferometer is said to be "in lock". During the first few minutes following the acquisition of a lock in any individual detector, the instrument typically experiences excess noise due to the ringing down of mechanical resonances in the mirror suspensions that were excited by impulsive forces applied during the lock acquisition procedure. After allowing for these resonances to damp down, the detector is placed into "science mode"; the data collected in science mode are available for gravitational wave searches. Science mode continues until the interferometer loses lock or becomes unstable for any reason. The gravitational wave strain signal (referred to in this paper as the gravitational wave data channel) is derived from the error signal of the feedback loop used to control the differential length of the interferometer arms. A 16 bit analog-to-digital converter is used to digitize the (uncalibrated) strain signal at a rate of $16384 \mathrm{~Hz}$.

To calibrate the error signal, the response to a known differential arm strain is measured, and the frequency-dependent effect of the feedback loop gain is measured and compensated for. The laser wavelength and the amplitude of the mirror drive signal required to move the interference pattern through a fixed number of fringes are used to calibrate the absolute scale for strain. The frequency response of the detector is determined via periodic swept-sine excitations of the end test masses. During detector operation, the calibration is tracked by injecting continuous, fixed-amplitude sinusoidal excitations into the end test mass control systems, and monitoring the amplitude of these signals at the measurement (error) point. The calibration procedure, and results, are described in more detail in [27, 28].

\section{B. The S1 run}

By the summer of 2002, all three LIGO detectors were operating reasonably stably and with reasonable in-lock duty cycle. As discussed below, the strain sensitivities of all three detectors were far from their design goals, but were nonetheless sensitive to gravitational wave bursts from energetic events in 


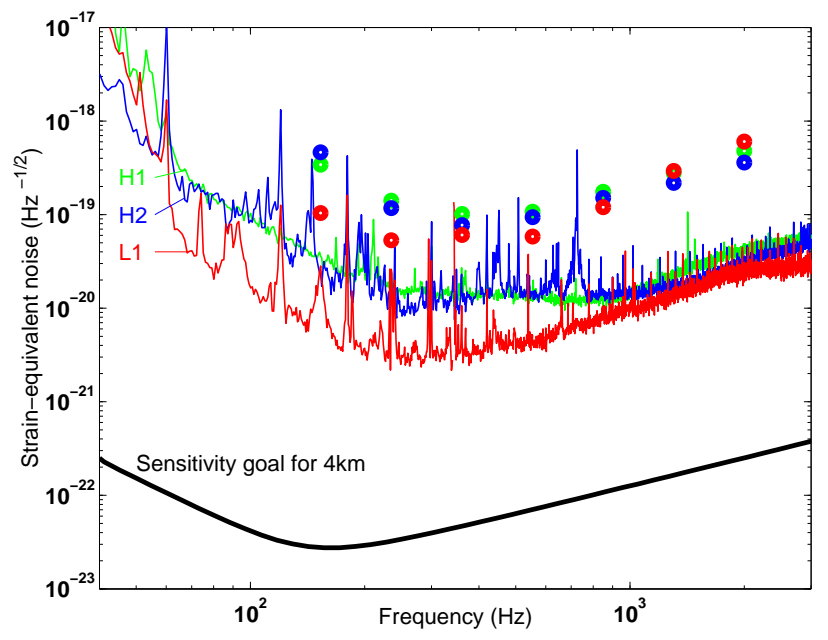

FIG. 1: Typical sensitivities of the three LIGO detectors during the $\mathrm{S} 1$ data run, in terms of equivalent strain noise amplitude density. The points are the root-sum-square strain $\left(h_{r s s}\right)$ of sine-Gaussian bursts for which our TFCLUSTERS analysis pipeline is $50 \%$ efficient, as reported in section $\nabla \mathrm{B}$

our Galactic neighborhood. The LIGO Laboratory decided that it was an appropriate time for the first Science Run, S1.

The $\mathrm{S} 1$ run consisted of a 408 hour continuous period from August 23 through September 9 of 2002, during which data were collected from all three LIGO interferometric detectors. The state of each of the detectors and the quality of the data being logged was continuously monitored through automated and manual procedures. As discussed above, in order to be sensitive to gravitational waves, the detectors must be in science mode. Environmental disturbances and various instrumental instabilities make it impossible to maintain lock at all times, reducing the effective observation time of the run. During $\mathrm{S} 1$, the science mode duty cycles of the three detectors were $41.7 \%$ for $\mathrm{L} 1,57.6 \%$ for $\mathrm{H} 1$, and $73.1 \%$ for $\mathrm{H} 2$. The burst search reported here makes use of the data when all three detectors were in science mode simultaneously, comprising 95.7 hours, or $23.4 \%$ duty cycle.

The strain sensitivity of the LIGO detectors is a strong function of gravitational wave frequency. In this analysis, we focus on a "detection band" of best strain sensitivity, from 150 to $3000 \mathrm{~Hz}$. Figure 1 shows amplitude spectra of strainequivalent noise, typical of the three LIGO detectors during the $\mathrm{S} 1$ run. The LIGO design strain sensitivity is also indicated for comparison. The differences among the three spectra reflect differences in the operating parameters and hardware implementations of the three instruments; they are in various stages of reaching the final design configuration. All detectors operated during S1 at lower effective laser power levels than the eventual level of $6 \mathrm{~W}$ at the interferometer input. Other major differences between the S1 state and the final configuration were partially implemented laser frequency and amplitude stabilization systems and partially implemented alignment control systems. Because of these conditions, the strain sensitivities of the three detectors were far from the design sensitivity (see Figure 1).

\section{Data preselection}

The data processing pipeline described in section Пmakes use of many adjustable parameters that can be tuned to optimize the search effectiveness. We performed these optimizations on a subset of the S1 data that was reserved exclusively for the purpose, and then not used further in the generation of scientific results. We called this reserved data set the "playground" data set. It was chosen to be about $10 \%$ of the total available triple coincidence data. The choice of which data to include was made by hand, to include as much variety of data quality as possible. The same playground data set was used for both the burst search and the search for inspiralling binary neutron stars [12]. This tuning procedure is described in section III Further, the data processing pipeline analyzed triplecoincidence data in six-minute stretches, for convenience in data handling. Lock stretches that were less than six minutes long, or data in the last $<6$ minutes of a longer lock stretch, were excluded from further analysis. After exclusion of the playground data and these lock stretch boundaries, 80.8 hours of triple-coincidence data remain.

Much effort has gone into improving the stationarity of the statistical properties of the detector noise, and understanding the noise fluctuations. However, both the detectors' responses, and their noise levels, were far from stationary, largely because the control systems were not yet completely implemented. In order to ensure that the data used for this burst search are of the highest available quality, we excluded locked stretches in which the noise in the gravitational wave channel exceeded a pre-determined threshold. The band-limited root-mean-square (BLRMS) noise power in the gravitational wave channel was monitored continuously in four bands (320 $-400 \mathrm{~Hz}, 400-600 \mathrm{~Hz}, 600-1600 \mathrm{~Hz}$, and $1600-3000$ $\mathrm{Hz}$ ). Whenever the BLRMS over a six-minute interval for any detector in any of these bands exceeded a threshold of 3 times the 68th percentile level for the entire run (10 times for the $320-400 \mathrm{~Hz}$ band), the data from that six-minute period were excluded from further analysis. A total of 54.6 hours of triple-coincidence data remains after this "BLRMS cut". A sufficiently strong gravitational wave burst could trigger the BLRMS cut and thereby prevent its own detection; the required amplitude is calculated in section $\nabla \mathrm{B}$

As discussed in section to gravitational waves was tracked by injecting sinusoidal calibration excitations into the end test mass control systems. Due to technical difficulties, these calibration lines were not reliable or available during some data taking periods. In order to ensure that all the data used in this search represent observations from detectors with well-understood response, data that show no, or anomalously low, calibration lines were excluded from further analysis (the "calibration cut"), leaving 35.5 hours of triple-coincidence data remaining. This is the final data sample used to search for gravitational wave bursts. 


\section{THE DATA PROCESSING PIPELINE}

In the analysis presented here, the purpose of the data processing pipeline is to identify candidate gravitational wave events in the data from all three detectors in coincidence. In this section, we discuss the procedures and algorithms used to identify coincident burst event candidates, the tuning of the most important parameters, and the procedures used to estimate the accidental coincident burst event rate. The entire analysis procedure, parameter tuning, event property estimation, and all other optimizations were developed using the playground data (section $\amalg \mathrm{C}$, and frozen before applying the analysis to the full S1 data set. In the process of analyzing the full data set, it became clear that many of the procedures and tunings were less than optimal, for a variety of reasons. We present the results of this first analysis in this paper, and intend to apply improved methods and optimizations (see section VIIB to the analysis of future data sets (which will have much greater sensitivity to gravitational wave bursts).

\section{A. Pipeline overview}

Figure 2 shows, in graphical form, the data processing pipeline used in this analysis. Most of the figure is used to schematically illustrate various steps in the pipeline of one of the interferometric detectors $(\mathrm{H} 1, \mathrm{~L} 1$, or $\mathrm{H} 2$, generically referred to here as IFO-1). The analysis pipelines of the other two IFOs (IFO-2 and IFO-3) are not shown in detail because they are identical to the first. The first step in the pipeline ("Band limited RMS \& calibration cuts") validates the strain channel data used in the analysis; only validated data (section IIC taken at times when all three detectors were operating simultaneously in science mode are used in this analysis. This step establishes the accumulated observation time, or livetime, for the analysis.

The next steps in the pipeline ("Prefiltering \& whitening") take as input the raw gravitational wave channel data from each detector, and prefilter the data stream (section ЩВ). The following step ("Burst event trigger generation") searches for bursts in the filtered data stream using two different burst detection algorithms (section $\amalg$, resulting in a set of event triggers at each detector. All data were processed in nonoverlapping segments that were six minutes long.

Our pipeline allows for the elimination of event triggers that are coincident in time with anomalous events in auxiliary channels that monitor the detector and the environment (see "Auxiliary channels" path and "Single IFO analysis" gate in Fig. 2]. The consideration of these potential vetoes will be described in section IIIE

Real gravitational wave bursts will cause a nearly simultaneous response in all three detectors, so in the next step we require temporal coincidence of single-detector event triggers ("Multi-IFO analysis" block in Fig. 2). We select as "Event candidates" only those combinations of single-detector event triggers that are consistent with originating from a single plane gravitational wave burst incident on the detector array (section IIIF).
Event triggers from the single-detector steps of our pipeline are mostly due to detector noise. The independence of noise triggers at the two LIGO sites is an important assumption in this analysis. The largest sources of noise above $100 \mathrm{~Hz}$ are generated internal to the detectors and are thus uncorrelated. Environmental disturbances can produce bursts of noise that cause triggers, and these can be coincident between the two sites within the $\pm 10 \mathrm{~ms}$ gravitational wave travel time if they propagate electromagnetically. Auxiliary sensors (e.g., magnetometers) monitor the environment and their inter-site correlations have been studied. Inter-site disturbances are calculated not to be important until the detectors are close to design sensitivity, and our studies of S1 data have not found evidence for coincident noise bursts even for $\mathrm{H} 1-\mathrm{H} 2$ where the coincident location increases the susceptibility to environmental disturbances. There does exist some coherence between the gravitational wave channels from the three detectors at certain frequencies [14], but there is no evidence that this contributes to coincident noise bursts.

Gravitational wave burst events detected at the two LIGO sites will be correlated in time. We can evaluate the mean rate of background events by measuring the mean rate of events that pass our coincident step after we have artificially shifted in time all the event triggers identified in one of the detectors, for example, L1. This background rate estimation is described in section IV

Finally, to determine the efficiency of the data processing pipeline to the detection of strain events incident on the detector array we add simulated events, of varying waveform and amplitude, to the input data stream and measure the fraction identified as event triggers in each detector. Knowing the detectors' sensitivity to gravitational waves incident from different directions we can combine the results of these simulations to determine the mean efficiency for detection of gravitational wave burst events incident on the detector array. The efficiency determination is described in section $\nabla$

\section{B. Prefiltering}

The event trigger generators we employ are designed to process data with a white noise spectrum (constant power spectral density as a function of frequency). The raw gravitational wave data from all three detectors during $\mathrm{S} 1$ are strongly colored, consisting essentially of randomly fluctuating noise with a strongly frequency-dependent power spectrum. These data can be converted to a noise equivalent strain signal through a response function which is also strongly frequency-dependent, and which is determined through the calibration procedure described in section III The noise also contains unwanted features such as spectral lines associated with interference from the $60 \mathrm{~Hz}$ power mains, mechanical resonances in the detector components, and other imperfections.

For the analysis presented here, the data from the gravitational wave data channel are passed through a linear filter, consisting of a 6-th order Butterworth high-pass filter with $150 \mathrm{~Hz}$ cut-off frequency to suppress large noise fluctuations 


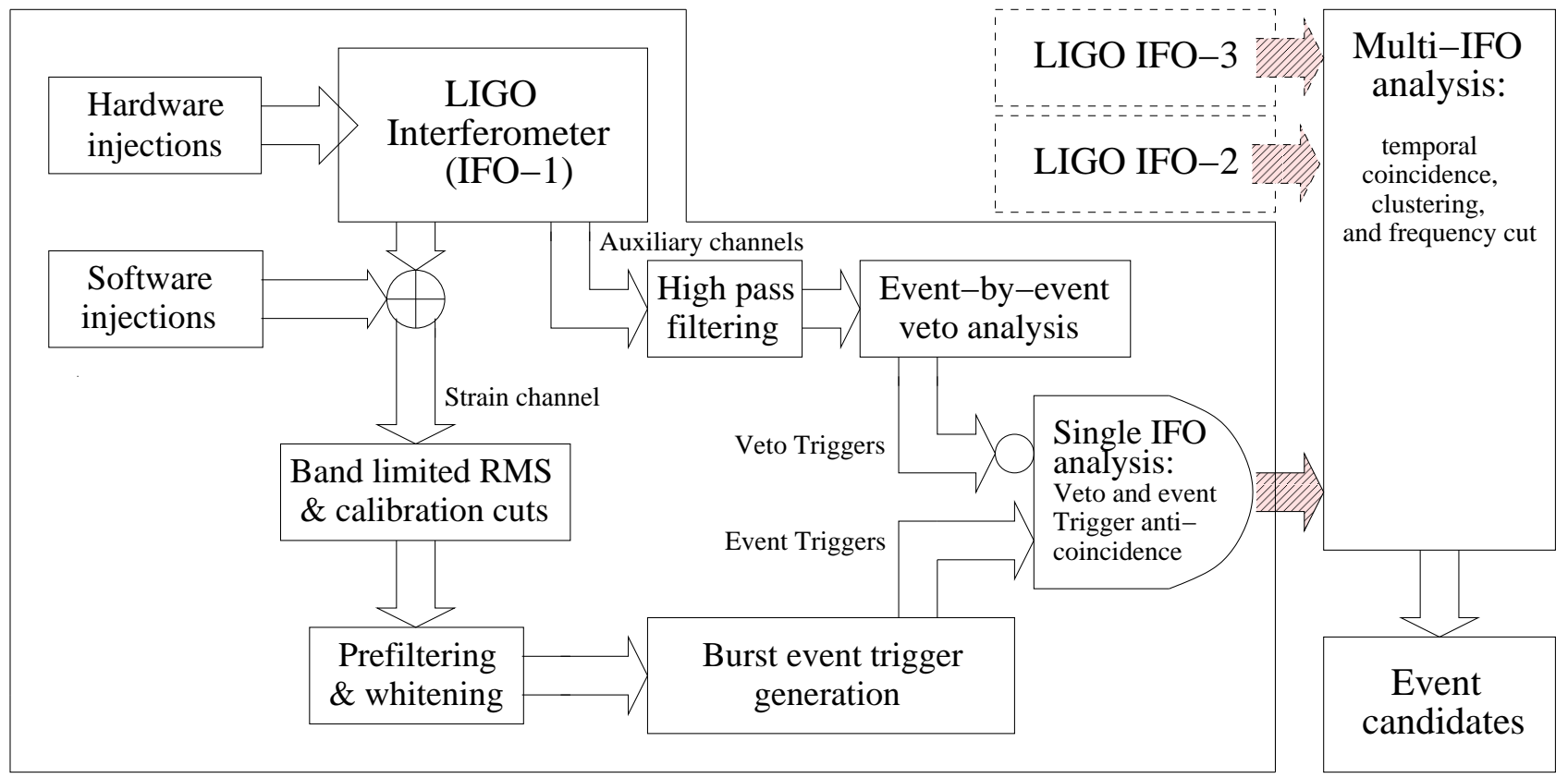

FIG. 2: Schematic outline of the S1 bursts analysis pipeline.

which were apparent at lower frequencies, and a whitening filter to flatten the noise spectrum at frequencies above 150 Hz. Because of the high-pass filtering, we are insensitive to Fourier components of a gravitational wave burst below 150 Hz. The whitening filters are determined using data taken just prior to the S1 run, and are different for each of the three detectors. No attempt has been made to incorporate the variation of the noise power with time, or to otherwise optimize the filtering. Further, no attempt has been made to remove spectral lines from the data stream or suppress their effect on the event trigger identification. It is likely that such pre-processing will be necessary for future, more sensitive searches with LIGO data [29].

The impulse response of the prefilter used for this analysis has a strong ringing, extending to $40 \mathrm{~ms}$. As a result of this ringing, the event trigger generation algorithms recognize an impulsive event in the strain channel as a cluster of events over a long period of time compared to both the sample rate and the light travel time between the detectors. This has important consequences for the event trigger time resolution and the time coincidence of event triggers generated in different detectors, as described in section [IIF] below [29].

\section{Event Trigger Generation}

We use two different techniques to identify event triggers from the prefiltered gravitational wave data channel at each detector. One technique, which we refer to as SLOPE, is based on Refs. [30, 31]. The second technique, which we refer to as TFCLUSTERS, is described in Refs. [32, 33]. SLOPE and TFCLUSTERS are two different approaches to identifying and selecting infrequent transient events that do not share the sta- tistical characteristics of detector noise and thus might be of gravitational wave origin. These algorithms are implemented within the LIGO Data Analysis System (LDAS [34]) environment.

The following discussion of the SLOPE and TFCLUSTERS event trigger generators describes and specifies the parameters that can be adjusted in order to optimize the performance of the algorithms. Some of the parameters can be established without reference to the data, since they effectively determine the response of the algorithm to the duration ( $4 \mathrm{~ms}$ to 100 $\mathrm{ms}$ ) and frequency band (150 to $3000 \mathrm{~Hz}$ ) characteristics of the bursts that are targeted in this search. Others have been optimized using the playground data defined in section $\amalg \mathrm{C}$ It is assumed that no (or very few) real gravitational wave bursts were present in the playground sample. All parameters were fixed prior to the processing of the full data set, in order to minimize the chance of bias in event trigger generation.

The parameter optimization, especially the choice of thresholds, is guided by competing demands. Lower thresholds on excess power or amplitude variations result in higher rates of event triggers caused by noise fluctuations, but also result in higher sensitivity to gravitational wave bursts. The criterion we adopted consists of minimizing the upper limit for a suite of simulated gravitational wave bursts, described in section $\nabla$ This minimization was applied to the playground data set where no triple coincidence event was found after the thresholds were fixed. This was consistent with a goal of obtaining a total number of accidental coincident triggers of order unity, when extrapolated to the remaining $90 \%$ of the full S1 data set.

Nevertheless, the playground data did not adequately represent the full S1 data set, and a variety of additional effects (including the ringing in the prefiltering, as discussed in section 
IIIB resulted in imperfect optimization of the data processing pipeline for both event trigger generators [29]. Therefore, the resulting number of estimated accidental coincidence events was somewhat larger than one, as discussed in section IVC and Table!

\section{SLOPE}

The SLOPE algorithm identifies candidate gravitational wave bursts via a threshold on the output of a linear filter applied to the prefiltered gravitational wave data in the time domain. We choose a filter that is essentially a differentiator (in time), and trigger on a slope in the data stream which is (statistically) inconsistent with expectations from white Gaussian noise. The SLOPE algorithm is most sensitive when the detector noise in the strain channel is whitened.

The parameters of the SLOPE filter have been tuned so that its highest sensitivity is for bursts in which the signal amplitude is increasing linearly with time for ten data samples $(10 \times 61 \mu \mathrm{sec})$. The response of the filter to sine waves rises with frequency from zero at DC, reaching its first and highest maximum at $1.1 \mathrm{kHz}$. Above this frequency, the response of the filter falls off, passing through several zeros and secondary maxima. Its $3 \mathrm{~dB}$ bandwidth is about $1.4 \mathrm{kHz}$ [29].

The filter output is searched for extrema indicating the presence of bursts. The peak search algorithm compares each successive filter output value with a threshold. If a filter output value is found to exceed the threshold, then that point and some number of output filter value after the first point exceeding the threshold are further analyzed. For the analysis considered here, 49 output filter values including the point that passed threshold are examined, a time interval of $3.0 \mathrm{~ms}$. The output filter value having the highest value in this time interval generates a single trigger. The amplitude of the trigger and time of the trigger are written to a trigger database. For this analysis, the threshold was fixed and did not adapt to changing noise levels [29].

\section{TFCLUSTERS}

The TFCLUSTERS event trigger generator is a detection algorithm which identifies connected regions (clusters) in a time-frequency plane where the power is not consistent with the expectations for stationary, colored Gaussian noise. The TFCLUSTERS algorithm is described in detail in [33], and various aspects of its implementation for real data are discussed in [32]. The implementation of TFCLUSTERS used for our analysis is described below.

The data from a six minute long segment are first prefiltered as described in section is constructed from 2880 periodograms calculated from 125 $\mathrm{ms}$ long non-overlapping subsegments of the six minute long segment [29].

A first level of threshold is applied to the spectrogram, resulting in a high-contrast pixelization. 2880 different measurements of the power are available for every frequency band of the spectrogram. Processing one frequency band at a time, the power measurements are fit with a Rice distribution [35]. Given this fit to the data, the Rice distribution is integrated from a power $\eta$ to infinity, and $\eta$ is varied until the integral is equal to a certain pre-defined fraction $p$. All the pixels of the spectrogram with power larger than $\eta$ are then labeled as black pixels, while pixels below the threshold are labeled as white pixels. The procedure was repeated for all the frequency bins in the spectrogram. The number $p$ is called the black pixel probability: in the absence of signals, any pixel in the spectrogram has, to a good approximation, an equal and independent probability $p$ of being black, in each frequency band. Because of this procedure, the effective threshold for black pixels varies in response to changing detector noise levels; the threshold is "adaptive", as opposed to the fixed threshold employed in the SLOPE algorithm.

The black pixels are then clustered, to look for bursts of excess power in a limited region of the time-frequency plane. Two levels of clustering are used by TFCLUSTERS, based on a study of simulated bursts with varying waveforms. First, a cluster is defined as the set of all black pixels which has at least one black nearest neighbor (i.e., was touching a black pixel by an "edge") in the set. All clusters containing at least five pixels are declared significant in this analysis. Second, clusters which are not significant according to the latter criterion are paired together. If the clusters in a pair are closer to each other in the time-frequency plane than a certain distance threshold, the pair of clusters is declared significant.

Clusters satisfying the first clustering condition on the raw size of a cluster are counted as event triggers. For clusters satisfying the second clustering condition, generalized clusters are formed by linking all the clusters which satisfy the distance thresholds, and these generalized clusters are counted as event triggers. For each event trigger, the time and frequency intervals over which the cluster extends, the total amount of power in the cluster, and the number of pixels it contains, are stored in a database. The total power in each cluster is a measure of the signal-to-noise ratio for the burst event. It is calculated without reference to the response of the detector to gravational wave bursts, so its relationship to the strength of the burst depends on the detector and frequency band.

The black pixel probability $p$ is tuned as described above. The values are different for the three different detectors and vary from 0.02 to 0.05 . The total power in the cluster is required to exceed a pre-determined threshold in postprocessing; this is effectively a cut on the signal-to-noise ratio for the burst event. The threshold on the power is the same for all three detectors, in order to obtain rates for false (noise) triggers which are roughly the same for all three detectors.

\section{SLOPE and TFCLUSTERS event triggers}

Figs. 3 and 4 show histograms of SLOPE and TFCLUSTERS event triggers before and after the application of the BLRMS and calibration cuts described in section IC The horizontal axis in these histograms is a measure of the amplitude or power of the excess signal identified by the SLOPE 


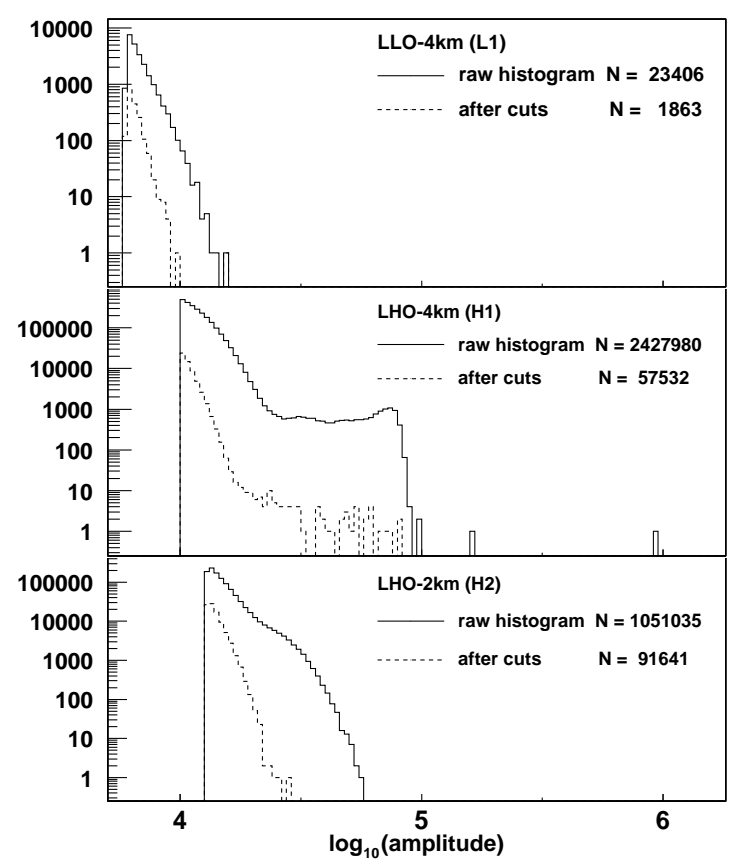

FIG. 3: Histogram of SLOPE event triggers from the three LIGO detectors, before and after the BLRMS and calibration cuts.

or TFCLUSTERS algorithms, respectively. These measures are indeed proportional to the true amplitude or power of a detected gravitational wave burst, as demonstrated in section V] However, no information about the detectors' calibrated response functions is used in forming these measures, so the proportionality constant is different for different waveforms, detectors, and data epochs (and is taken into account in the evaluation of the detection efficiency, section $\nabla$. The lower limits on the horizontal axis in these histograms correspond to the threshold applied to that event trigger for input into the next step in the data processing pipeline (triple coincidence).

\section{E. Auxiliary Channel Vetoes}

Environmental disturbances and detector instabilities could also produce event triggers. We collect data in a large number of auxiliary channels which monitor the detector and the environment, in order to look for time-coincident bursts and thus form vetoes for such false triggers. Our pipeline has the capability to search for such bursts in auxiliary channels, and veto an event trigger if it is time-coincident with such a burst. Engineering runs performed prior to the $\mathrm{S} 1$ run indicated that such vetoes could be very efficacious, reducing the rate of false event triggers with minimal loss of livetime, due to clearly identifiable instabilities in the detectors. However, once these instabilities were identified, they were eliminated through improved instrumentation, resulting in much improved stability during S1. After careful study, no vetoing criteria using auxiliary interferometer and physical environment monitor channels are found to be especially efficacious in the $\mathrm{S} 1$ data, for

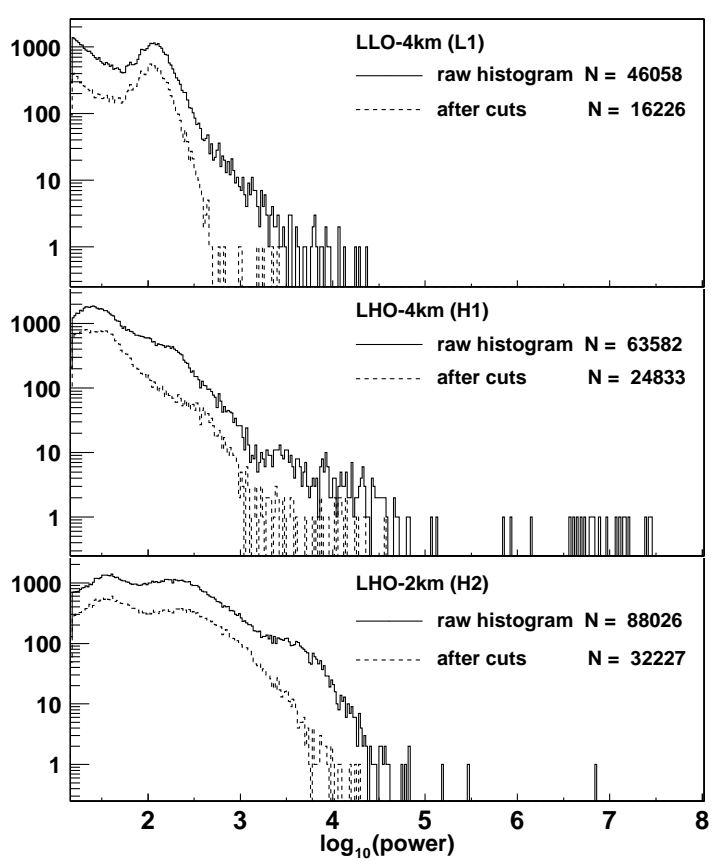

FIG. 4: Histogram of TFCLUSTERS event triggers from the three LIGO detectors, before and after the BLRMS and calibration cuts.

this burst search. The most promising vetoing channels in the S1 data are interferometer sensors that are closely related to the gravitational wave channel. While we investigated a number of such channels and methods for identifying veto criteria, in the end we concluded that further study was needed before any of these could safely be used to exclude data from analysis. Further, employing the identified vetoes would have made a negligible difference in the results of this analysis. Thus, in this analysis, we apply no vetoes based on auxiliary channels.

\section{F. Coincidence}

The final stage of our data processing pipeline brings together the event triggers generated by a particular event trigger generator (either SLOPE or TFCLUSTERS) and assembles a smaller list of coincident event trigger triplets. Each triplet consists of an event trigger from each detector that occur within an interval consistent with their origin in a single gravitational wave burst. These triplets are the event candidates that form the basis for our determination of bounds on the rate of gravitational wave bursts incident on the Earth.

Temporal coincidence is the most obvious application of coincidence for selection of gravitational wave events and exclusion of noise events. The LIGO detectors are approximately co-aligned and co-planar. As a result, they all sense approximately the same polarization of any incident gravitational wave. Correspondingly, all estimated parameters of the burst (such as strain amplitude and frequency band) should be, up to uncertainties in the estimation, the same for all three detectors (after accounting for the differences in the detectors' 
sensitivities). In the analysis presented here we require temporal coincidence (to an appropriate precision) for both the SLOPE and the TFCLUSTERS pipelines. Additionally, TFCLUSTERS events are also characterized by frequency information; we require consistency between the frequency bands in a coincident triplet (section IIIF3. No attempt is made to require coincident event triggers to have consistent amplitudes and waveforms [29].

In the remainder of this section we describe in greater detail the elements of the data processing pipeline coincidence step.

\section{Temporal coincidence}

Gravitational waves arrive at the Earth as plane waves. Since gravitational waves are assumed to propagate at the speed of light, the interval between event triggers in the different detectors should be no more than the greater of the light propagation time between the detectors and the uncertainty in the arrival time determination of a prototypical burst associated with the event trigger generator. Different timing uncertainties are associated with different event trigger generators. Correspondingly, we use different window durations for SLOPE and TFCLUSTERS. Given a window, we compare the start times of the event triggers generated in each of the three detectors. We form an event trigger triplet, or triplet for short, from all combinations of $\mathrm{H} 1, \mathrm{H} 2$ and $\mathrm{L} 1$ events whose start times all lie within the window duration.

As described in section 11 B the input to the event trigger generators is processed through a high-pass filter that rang strongly. As a result of this ringing, impulsive events lead to a train of multiple SLOPE triggers, with a total duration of approximately $40 \mathrm{~ms}$. We add $10 \mathrm{~ms}$ to this, corresponding to the light travel time between detectors, to determine a $50 \mathrm{~ms}$ window for temporal coincidence of SLOPE events [29].

As described in section IIIC2 TFCLUSTERS was tuned to a natural time resolution of $125 \mathrm{~ms}$, much larger than the light travel time between the detectors. On the basis of studies which indicated a larger range of trigger time differences for simulated signals, we expanded this and use a $500 \mathrm{~ms}$ window to determine triplets of temporally coincident TFCLUSTERS events [29].

\section{Clustering}

The next step in the multiple-detector coincidence analysis is to cluster the events from each detector (this is unrelated to the pixel clustering that forms the heart of the TFCLUSTERS event trigger generation, section $\amalg$ C2 . Both the TFCLUSTERS and the SLOPE event trigger generators often associate several event triggers with the same "burst" feature. For instance, the ringing of a $1 \mathrm{~ms}$ Gaussian due to the detector response and the prefiltering of the data (section [IIB) can produce several closely spaced event triggers. TFCLUSTERS often associates multiple triggers with the same broadband event, all with the same start time but different frequency. Since we are interested in the identification of time intervals where "something unusual" has happened simultaneously at multiple detectors, we want to cluster these sets of closely spaced events.

Clustering takes place only after the time coincidence step. We require a minimum separation in time between distinct coincident trigger triplets, of 0.5 seconds; triplets that are separated in time by less than this amount are clustered together into one clustered event triplet (event candidate). The choice of the clustering window is based on the study of noise triggers and simulated bursts (section $\nabla B$ ). In the TFCLUSTERS pipeline, 0.5 seconds is the width of the coincidence window between triggers from the three detectors. In the SLOPE pipeline, the coincidence window of $50 \mathrm{~ms}$ is too small a separation to avoid ambiguities in the definition of clusters and in the event counting, so we use 0.5 seconds for consistency with the TFCLUSTERS pipeline [29].

All triggers in the cluster are assumed to originate from one burst event. Guided by simulation studies (section $\nabla \mathrm{B}$, the start time, frequency band, and amplitude or power of the event is taken to be that of the trigger with the largest amplitude or power in the cluster.

\section{TFCLUSTERS frequency cut}

For TFLUSTERS we apply one more criterion in the coincidence step of the pipeline. A triplet of event triggers that arises from a single gravitational wave burst incident on all the detectors should have consistent values for the estimated parameters of the burst. TFCLUSTERS characterizes each burst event trigger by its bandwidth: the low and high frequency bound $\left(f_{\text {low }}, f_{\text {high }}\right)$ of the cluster identified in the timefrequency plane. When multiple triggers from one detector are clustered in time as described in section IIIF2 above, the inclusive frequency band for that clustered event trigger is formed. For TFCLUSTERS triggers only, we require that the frequency bands of the clustered event triggers from each detector in the triplet either overlap, or are separated in frequency space by no more than a fixed window of $\Delta f=80 \mathrm{~Hz}$, based on studies of the simulations described in section $\mathrm{VB}$

\section{BACKGROUND AND SIGNAL RATES}

The data processing pipeline (section 11 generates background event triggers originating in noise level fluctuations in the detectors, due to random processes or environmental or instrumental disturbances. Our primary means to reject such background event triggers is temporal coincidence between the three detectors in the LIGO array (section IIIF. To the extent that noise fluctuations in each of the detectors are random, uncorrelated, and follow Poisson statistics, the primary background comes from accidental coincident events, and the accidental triple-coincidence rate can be predicted from the observed instantaneous single-detector event rates. 


\section{A. Background estimation}

We have chosen to tune our event trigger generators (using the playground data sample) so as to produce an estimated accidental triple-coincidence rate of one event over the entire S1 observation time, as discussed in section

Again assuming no correlations between noise fluctuations in the three detectors, we can indirectly measure the rate of accidental triple-coincident events from triple-coincidence rates when artificial time shifts are introduced between singledetector event triggers.

Such time shifted triple-coincidence events are free of contamination from true gravitational wave bursts (assuming that such bursts are rare), and thus are an unbiassed estimate of the accidental triple-coincidence rate. The distribution in the number of time shifted triple-coincidence events should follow a Poisson distribution. These distributions can be fitted to obtain the expected number of background events for use in our statistical analysis.

The time shifts should be larger than the maximum duration of a real (noise-induced or gravitational wave-induced) detectable burst, or else the events will be correlated and will not obey Poisson statistics. The time shifts should also be shorter than the typical time scale over which the singledetector event rates vary substantially, so that the number of events for different time shifts will be Poisson distributed for a quasi-stationary process.

To establish a lower limit on the time shift required to ensure uncorrelated noise event triggers, we histogram the time delay between consecutive events in the three detectors, shown in Fig. 5 for the TFCLUSTERS event trigger generator (the distributions are similar for the SLOPE event trigger generator). The distributions of delay times follows the expected exponential form for delay times exceeding 8 seconds (vertical dashed lines), for all three detectors. Any residual auto-correlations present in the data will rapidly decay for delay times exceeding 8 seconds, and in the case of many $(N)$ time-shift experiments, their potentially biased contribution to the Poisson estimate reduce as $1 / N$.

The assumption that noise fluctuations are uncorrelated between detectors is questionable for the two detectors colocated at the Hanford site [36], H1 and H2. Indeed, there exists evidence for short-term, narrow-band correlations in the noise power between the $\mathrm{H} 1$ and $\mathrm{H} 2$ detectors associated with power line harmonics, as well as correlations between L1 and $\mathrm{H} 1$ or $\mathrm{H} 2$ associated with harmonics of the data acquisition buffer rate [14]. The power line harmonics integrate away over long time scales, and the data acquisition buffer rate harmonics only appear after long integration times. It is the short term correlations that concern us here. We have found no detectable evidence of short term correlated noise fluctuations associated with these sources of narrow-band correlations.

In order to account for any potential correlations in noise fluctuations between $\mathrm{H} 1$ and $\mathrm{H} 2$, we have performed our timeshifted coincidence measurements by shifting the time between event triggers found in the L1 data and those found in the $\mathrm{H} 1$ and $\mathrm{H} 2$ data, while keeping zero time shift between $\mathrm{H} 1$ and $\mathrm{H} 2$.

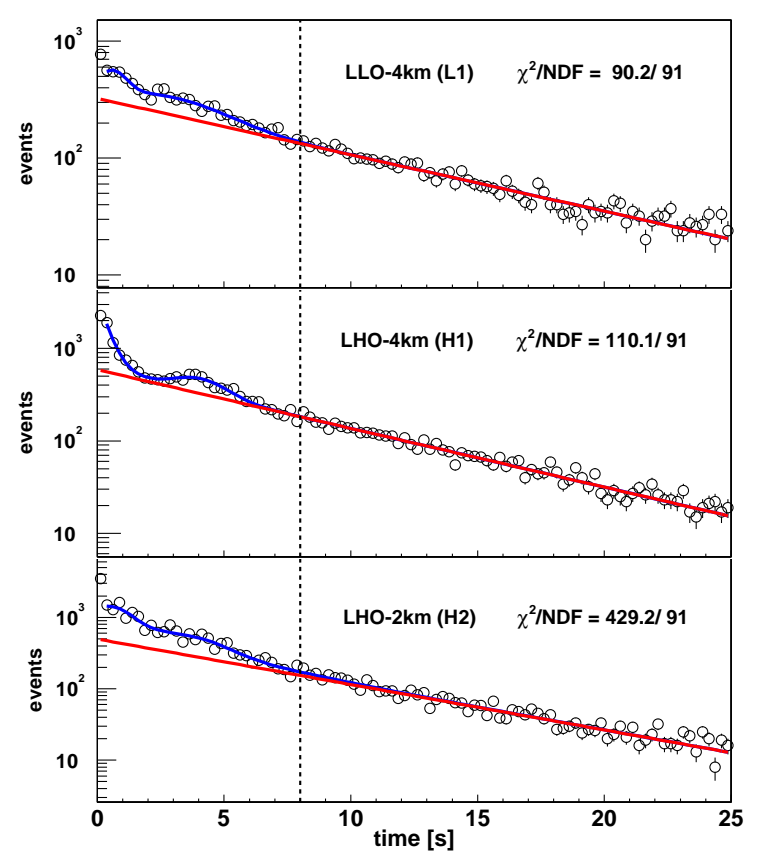

FIG. 5: Histograms of the time delay between consecutive events in the TFCLUSTERS event trigger generation, for the L1, H1, and $\mathrm{H} 2$ detectors. The curves are components of fits to the distributions that incorporate the expectations for short time delay correlations and long time delay random, uncorrelated events. The vertical dashed lines indicate the time delay beyond which consecutive events are consistent with being uncorrelated.

We have performed multiple time-shift experiments with the SLOPE and TFCLUSTERS event trigger generators between the Livingston and Hanford sites. The resulting number of time-shifted triple coincident events from 24 such experiments in the $[-100,100]$ second range with 8 second steps are shown in Figs. 6 and 7 for TFCLUSTERS and SLOPE, respectively. The distributions of background events for the 24 non-zero time shifts (lower plots in Figs. 6 and 7) are fitted with Poisson predictions and are found to be consistent with the expectation from Poisson statistics. Averages and Poisson mean values for different step and window sizes vary by less than 0.5 events.

In estimating the background rate, we have considered time shifts between 8 seconds (to avoid correlated events; see Fig. 5] and 100 seconds (to minimize dependence on any non-stationarity in the instantaneous event rate). These timeshift measurements yield estimates of the number of accidental triple-coincidence (background) events $\mu_{B}$ for the TFCLUSTERS and SLOPE event triggers. Because these measurements can be made with many, statistically-independent time shifts, the resulting statistical errors on these estimates are small.

The presence of any remaining non-stationarity in the background event rate, however, will result in errors in the background rate estimation. In fact, the instantaneous event trigger rate is observed to vary for both event trigger generators. The 

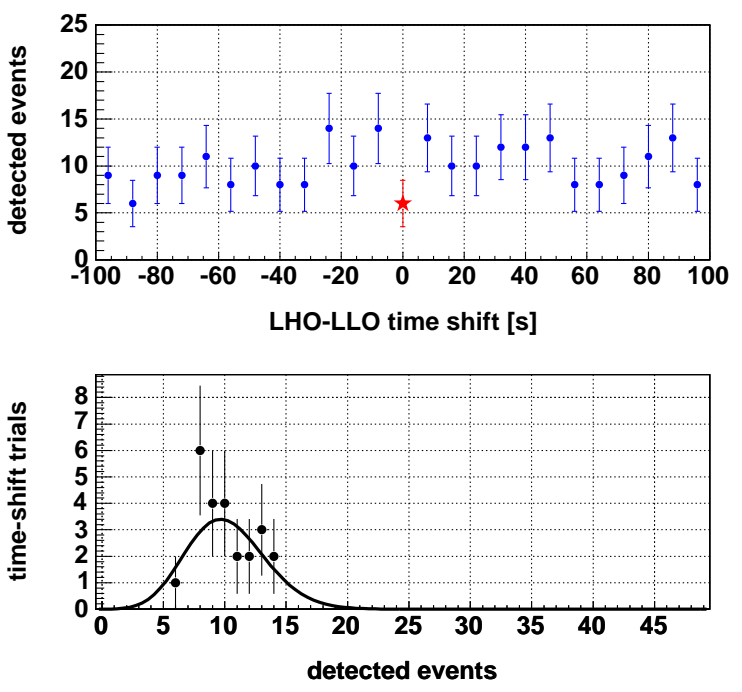

FIG. 6: Time-shifted triple coincident events from TFCLUSTERS event triggers, as a function of an artificial time shift introduced between the Hanford (LHO) and Livingston (LLO) sites. Top: Number of events versus time shift, in 8 second steps; the point at zero time shift is the number of true triple coincident events. Bottom: Histogram of the number of time-shifted coincident events, with the Poisson fit overlaid (the zero time shift point is excluded). In both plots, the error bars are Poissonian.
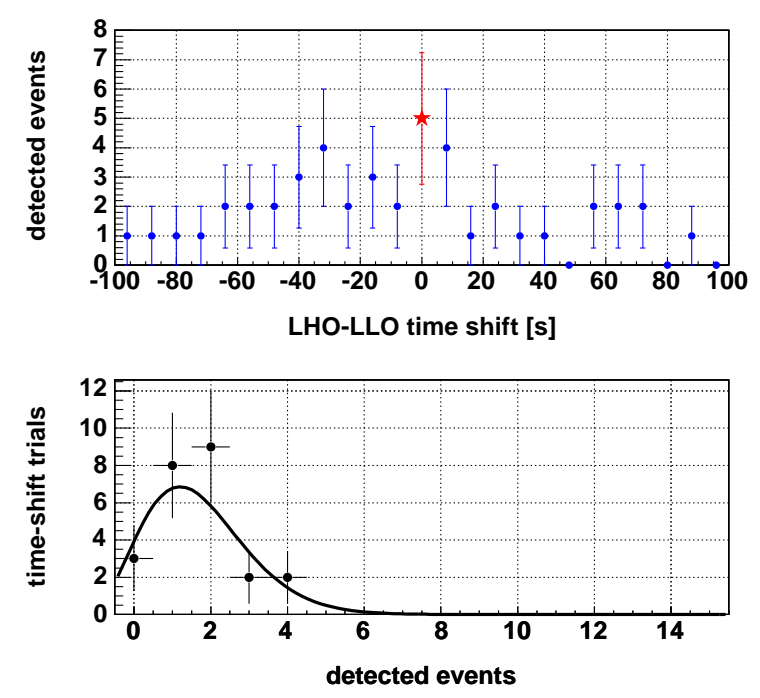

FIG. 7: Same as Fig. 6 but for the SLOPE event trigger generator.

variability of the trigger rate is sufficiently severe for SLOPE that the background rate estimation is deemed unreliable. Because of the fixed (non-adaptive) threshold employed in the SLOPE algorithm, the trigger rate of the individual interferometers varies by more than a factor of one thousand, sometimes on timescales of 10 seconds or less. All five events of the zero time lag coincidences and most of the time-shifted coincidences in Fig. 7 come from a single 360-second segment, corresponding to a coincidence of data segments from three interferometers with very high burst singles rates. Even within that segment, the singles rate varies markedly, making it difficult to reliably estimate the background rate. For this reason, we choose not to use the SLOPE pipeline to set a limit on the rate of gravitational wave bursts [29].

It should be noted that before these coincidences and background rates were available, we decided to set our upper limits using the results from the event trigger generator which yielded the better efficiency for detecting gravitational wave bursts, as measured by our simulations. For almost all waveforms this turned out to be the TFCLUSTERS pipeline (see section VD. Thus, even if the background rate (and thus the rate of excess triggers) from the SLOPE pipeline could be reliably estimated, the primary results from this search would still be based on the TFCLUSTERS pipeline.

\section{B. Signal candidate estimation}

An excess in the number of coincident (zero-time-shift) events over the estimated background can be estimated statistically. Here we make use of the unified approach of Feldman and Cousins [37]. This approach provides an unambiguous prescription for establishing a statistical excess of signal candidate events at a specified confidence level (that is, a lower limit to the confidence interval that is greater than zero). However, as discussed in section \ we have not yet characterized our detectors and data analysis procedures sufficiently well to claim that any such excess is a detection of gravitational wave bursts. We therefore use only the upper endpoint on the confidence interval for the number of signal candidate events to set an upper limit on the rate of gravitational wave bursts.

Starting from an observed number of events $n$ and an estimate of the number of background events $\mu_{B}$, we build confidence bands for the number of signal events $\mu_{S}$ according to the formula:

$$
\sum_{n_{0}=n_{1}}^{n_{2}} p\left(n_{0}\right)_{\mu_{S}+\mu_{B}} \geq \alpha
$$

where $p(n)_{\mu_{S}+\mu_{B}}$ is the Poisson probability density function

$$
p(n)_{\mu_{S}+\mu_{B}}=\left(\mu_{S}+\mu_{B}\right)^{n} \frac{e^{-\left(\mu_{S}+\mu_{B}\right)}}{n !} .
$$

The sum extremes, $n_{1}$ and $n_{2}$, are chosen according to a likelihood ranking principle [37]. In our implementation, we assume both signal and background are Poisson distributed. We report confidence bands for $\alpha=90 \%, 95 \%$, and $99 \%$.

We account for the statistical error on the background estimation following the method described in [38, 39], where a Gaussian background uncertainty is folded in the formulation of the probability density function. We replace $p(n)_{\mu_{S}+\mu_{B}}$ in Eqn. 4.1 with

$$
q(n)_{\mu_{S}+\mu_{B}}=\frac{1}{\sqrt{2 \pi} \sigma_{B}} \int_{0}^{\infty} p(n)_{\mu_{S}+\mu_{B}^{\prime}} e^{-\frac{\left(\mu_{B}-\mu_{B}^{\prime}\right)^{2}}{2 \sigma_{B}^{2}}} d \mu_{B}^{\prime}
$$

where $\sigma_{B}$ is the estimated background error. This marginalization, performed through a Monte Carlo calculation, is used 
in the construction of confidence bands for the estimated background $\mu_{B} \pm \sigma_{B}$.

\section{Event rate bound}

Table I shows the number of coincident events, the estimated number of accidental coincident events (background), and the confidence bands that we find at the $90 \%, 95 \%$, and $99 \%$ confidence levels on the number of excess events and the event rate (over 35.5 hours of S1 observation time), using the TFCLUSTERS event trigger generator pipeline. The upper bounds of the confidence bands are taken to be the upper limit on the number of signal events, at that confidence level. At the $90 \%$ confidence level, the search yields an upper limit of 2.3 events in 35.5 hours. As discussed in section[IVA because of the variability of the event trigger rate in the SLOPE pipeline, we choose not to use it to set a limit on the rate of gravitational wave bursts.

TABLE I: Confidence bands on the number of excess events in the $\mathrm{S} 1$ run (35.5 hours of observation time) from the TFCLUSTERS pipeline.

\begin{tabular}{lc}
\hline \hline Coincident events & 6 \\
Background & $10.1 \pm 0.6$ \\
$90 \%$ confidence band & $0-2.3$ \\
$95 \%$ confidence band & $0-3.5$ \\
$99 \%$ confidence band & $0-5.9$ \\
\hline \hline
\end{tabular}

Given the estimated backgrounds from the time shift analyses, the number of TFCLUSTERS events at zero time lag is somewhat low (Table \and Fig. 6. None of the events detected by SLOPE were detected by TFCLUSTERS. This is not in itself surprising, since the two event trigger generators have different sensitivities to different waveforms, but it does indicate that none of the events were far above threshold for that trigger generator, since the largest differences in efficiency between the two event trigger generators was approximately a factor of 6 (section $\mathrm{VD}$ below). The probability of obtaining six or fewer TFCLUSTERS events, given our estimated background, is approximately $12 \%$. We found no reason to suspect any systematic errors in our background estimate for this pipeline. Alternative methods of estimating the background (simple estimates based on the average singles rates and the coincidence window, time shift analyses where all three detectors are shifted as opposed to holding $\mathrm{H} 1-\mathrm{H} 2$ fixed at zero delay) did not give significantly different background rates.

\section{EFFICIENCY DETERMINATION}

In order to interpret our bound on the observed rate for coincident gravitational wave bursts, we study the response of the LIGO detectors and our analysis pipeline to simulated signals with varying waveforms, durations, bandwidth, and peak amplitudes. The simulated signals were injected into the gravitational wave data stream from each of the three detectors, as far upstream in the pipeline as was practical (after data acquisition and ingestion into the LIGO Data Analysis System).

The same data that were used to search for coincident bursts (section IIC) were also used for these simulations; and, for the purposes of these simulations, these data are assumed to consist entirely of noise (no real gravitational wave bursts present). Approximately $20 \%$ of the S1 data was used for these simulations, spanning the entire data run uniformly, in order to fairly represent the noise and detector sensitivities throughout the run.

We present the results for the efficiency determinations for both TFCLUSTERS and SLOPE event trigger generators, even though (as noted in section IVA we do not use SLOPE to derive a final limit on the rate of gravitational wave bursts.

\section{A. Waveforms}

The astrophysical origin, and waveform morphology, of the gravitational wave bursts we search for in this work are a priori unknown. A broad range of signal waveforms were considered. These include astrophysically-motivated waveforms, such as the results of supernova simulations [40, 41], as well as ad-hoc waveforms such as Gaussians, damped sinusoids, sine-Gaussians, Hermite-Gaussians, and others. Guided by the simulations in [40, 41], we have endeavored to be sensitive to any waveform that adds excess power (over that of the detector noise) in the LIGO S1 sensitivity band (150 to 3000 $\mathrm{Hz}$ ), with durations between $4 \mathrm{~ms}$ and $100 \mathrm{~ms}$.

In order to evaluate our sensitivity to such bursts, we must model the waveforms in some general way. For the results presented here, we have chosen to focus on two classes of ad hoc waveforms, which we regard as "surrogates" for real astrophysical signals. The first are broad-band, limited-duration Gaussians of the form

$$
h\left(t+t_{0}\right)=h_{0} \exp \left(-t^{2} / \tau^{2}\right),
$$

with varying peak amplitude $h_{0}$, peak time $t_{0}$, and duration $\tau$ (Fig. 8). The second are narrower-band, limited-duration sine-Gaussians of the form

$$
h\left(t+t_{0}\right)=h_{0} \sin \left(2 \pi f_{0} t\right) \exp \left(-t^{2} / \tau^{2}\right) .
$$

The duration of the sine-Gaussians were chosen to be $\tau=$ $2 / f_{0}$. Their Fourier transforms $\tilde{h}(f)$ span a (Gaussian) frequency band of $\sigma_{f}=f_{0} / Q$ centered about the central frequency $f_{0}$, where $Q \equiv f_{0} / \sigma_{f}=\sqrt{2} \pi \tau f_{0} \simeq 8.9$. We have chosen eight different central frequencies, spaced logarithmically, and spanning the LIGO sensitivity band: $f_{0}=$ [100, 153, 235, 361, 554, 849, 1304, 2000] Hz (Fig.9).

Our analysis pipeline detection efficiency depends on the burst duration, frequency band, and some measure of burst "strength"; it does not depend strongly on the precise waveform morphology. In order to facilitate comparison of the burst strength with the detectors' equivalent strain noise, and with burst waveforms having similar properties but different detailed morphologies, we define two useful measures of the burst strength. The root-sum-square (rss) amplitude spectral 

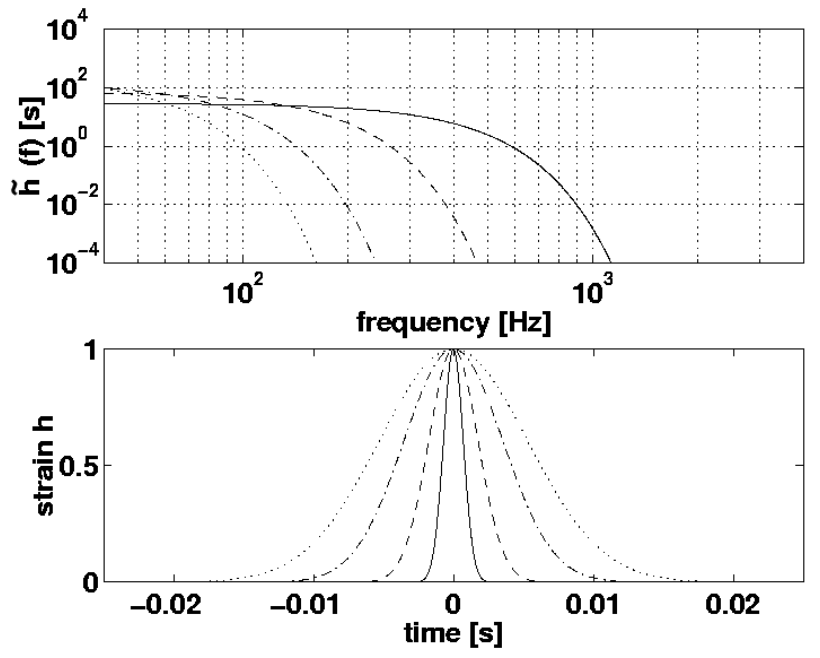

FIG. 8: Gaussian waveforms, with varying duration, as described in section $\nabla \mathrm{A}$ Top: Frequency spectrum. Bottom: Time series.
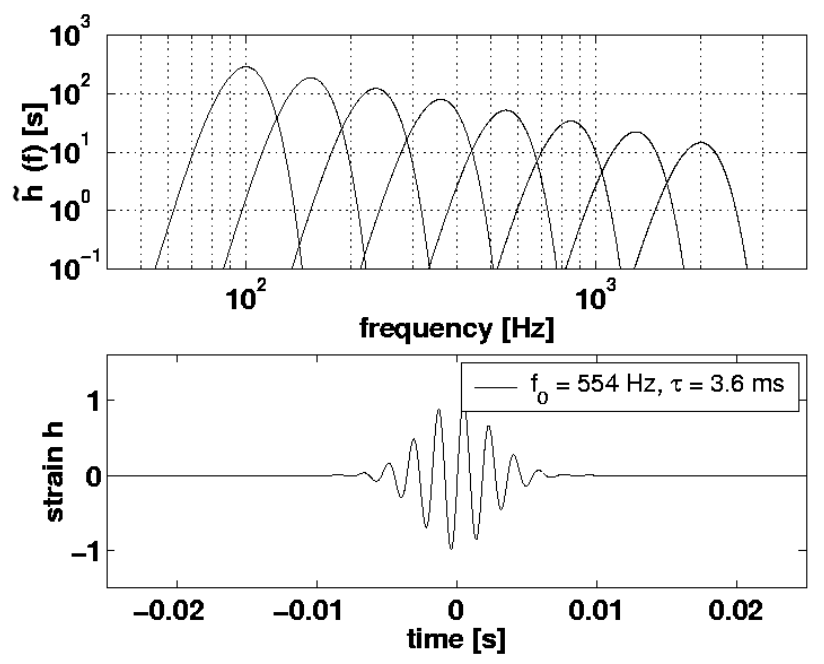

FIG. 9: Sine-Gaussian waveforms, with central frequency $f_{0}$ evenly spaced in $\log (\mathrm{f})$, and $Q \sim 9$, as described in section $\nabla \mathrm{A}$ Top: Frequency spectrum. Bottom: Time series for a sine-Gaussian with $\mathrm{f}_{0}=554 \mathrm{~Hz}$.

density for such bursts, in units of dimensionless strain per root $\mathrm{Hz}$, is defined by:

$$
\begin{aligned}
h_{r s s} & \equiv \sqrt{\int|h|^{2} d t} ; \\
& =\sqrt{\sqrt{\pi / 2} \tau} h_{0}(\text { Gaussians)} ; \\
& =\sqrt{Q /\left(4 \sqrt{\pi} f_{0}\right)} h_{0} \text { (sine-Gaussians) } .
\end{aligned}
$$

The characteristic strain amplitude, in units of dimensionless strain, is defined by [42]:

$$
\begin{aligned}
h_{\text {char }} & \equiv f_{c}\left|\tilde{h}\left(f_{c}\right)\right| \\
& =\sqrt{\pi}\left(f_{c} \tau\right) \exp \left(-\left(\pi f_{c} \tau\right)^{2}\right) h_{0} \text { (Gaussians);(5.7) }
\end{aligned}
$$

$$
=\sqrt{\pi}\left(f_{c} \tau / 2\right) h_{0}\left(\text { sine-Gaussians, } f_{c}=f_{0}\right) .
$$

Here, $\tilde{h}(f)$ is the Fourier transform of $h(t)$, defined by

$$
\tilde{h}(f)=\int_{-\infty}^{\infty} h(t) e^{-i 2 \pi f t} d t
$$

and $f_{c}$ is a characteristic frequency (typically, either the frequency at which $\tilde{h}(f)$ peaks, or the frequency where the detector is most sensitive). For our sine-Gaussians, we choose $f_{c}$ to be the central frequency $f_{0}=2 / \tau$; for Gaussians, we choose $f_{c}$ to be the frequency at which all three LIGO detectors had approximately best sensitivity during S1, $f_{c} \simeq 300$ Hz (see Fig.1).

\section{B. Simulations}

In order to add the simulated signal (in units of dimensionless strain) to the raw detector data (in units of ADC counts), we must convert, or filter, the signal using the detector response function (in counts per strain) obtained through the calibration procedure described in section 1 The simulated signals, padded with zeros to minimize edge effects, are filtered through the detector response function in the Fourier domain, yielding a time series in ADC counts that can be added directly to the raw gravitational wave data stream at the beginning of the data processing pipeline. These simulated signals can be injected at any chosen point in time, and with any chosen amplitude. The uncertainty in the calibration information is the largest source of systematic error in this analysis (section VIC].

For each waveform, we evaluate the efficiency for detection through each of the three LIGO detectors and analysis pipelines, as a function of $h_{r s s}$ (defined in equation 5.3), assuming optimal wave direction and polarization. Approximately 80 simulations are performed for each combination of waveform, $h_{r s s}$, detector, and event trigger generator, using data spanning the S1 run. In Figs. 10 and 11 we plot detection efficiencies and average signal strengths for the TFCLUSTERS and SLOPE event trigger generators, respectively. Although our event trigger generators do not necessarily trigger on excess power, we find that the "strength" of the signal reported by either event trigger generator (the SLOPE "amplitude" or the square root of the TFCLUSTERS "power") is proportional to the actual amplitude of the injected signal over a broad range of $h_{r s s}$. This is illustrated by the lower plots in Figs.10 and 11(for one particular waveform). We emphasize that the "power" or "amplitude" that is plotted in Figs. 10 and 11 respectively (and in Figs. 4 and 3, are purely algorithmdependent quantities which are compared with thresholds to define event triggers; they are not designed to be true measures of the burst power or amplitude, and they will be different for different detectors and waveforms.

As expected, the efficiencies are essentially $100 \%$ for large values of $h_{r s s}$, consistent with noise and thus $0 \%$ efficiency for small $h_{r s s}$, and transitioning smoothly over a narrow intermediate range of $h_{r s s}$. The time window used to associate 
a TFCLUSTERS event trigger around the time of the injection $(0.5 \mathrm{~s})$ is larger than for a SLOPE event trigger, so the observed efficiency for small $h_{r s s}$ waveforms appears larger in Fig. 10 than in Fig. 11] The results in both cases are empirically found to be well fitted to simple sigmoid curves in $\log _{10}\left(h_{r s s}\right)$ :

$$
\varepsilon\left(h_{r s s}\right)=\frac{1}{1+\mathrm{e}^{-\left(\log _{10} h_{r s s}-b\right) / a}}
$$

where $b=\log _{10} h_{r s s 1 / 2}$ determines the strain per root $\mathrm{Hz}$ $h_{r s s 1 / 2}$ at which the efficiency is equal to $1 / 2$, and $a$ governs the width of the transition from 0 to 1 in $\log _{10}\left(h_{r s s}\right)$. It is specific to a given waveform, detector, event trigger generator, and data epoch. All fits resulted in good fit quality, except at the smallest values of $h_{r s s}$, where noise triggers dominate; we exclude such triggers from our definition of "efficiency", and use the sigmoid fits to extrapolate to zero efficiency at small values of $h_{r s s}$. Examples of sigmoid fits are shown in Fig.10 and Fig. 11

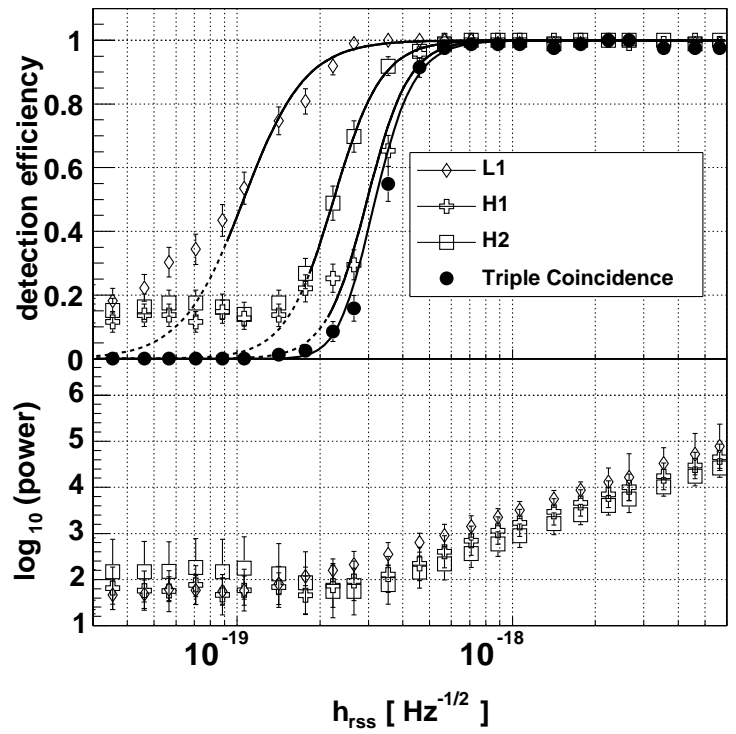

FIG. 10: The response of the TFCLUSTERS event trigger generator to Gaussian bursts with $\tau=1 \mathrm{~ms}$, embedded in $\mathrm{S} 1$ data, as a function of the root-sum-square strain $h_{r s s}$. Upper plot: Average burst detection efficiency. The efficiencies were evaluated through simulations of burst waveforms with optimal wave direction and polarization, injected into $\mathrm{S} 1$ data. The simulated data points are fitted to sigmoid curves, shown, in the region where the efficiency is not dominated by random noise triggers. The curve for the triplecoincidence is the product of the single-detector efficiency curves, and can be directly compared with the triple-coincidence simulation data points. Lower plot: Average detected signal strength for each of the three LIGO detectors.

The efficiencies shown in Figs. 10 and 11] do not remain at unity to arbitrarily high amplitudes. A sufficiently strong gravitational wave could trigger the BLRMS cut and in that way effectively prevent its own detection in this search. The most susceptible band for such a possibility is the 320-400

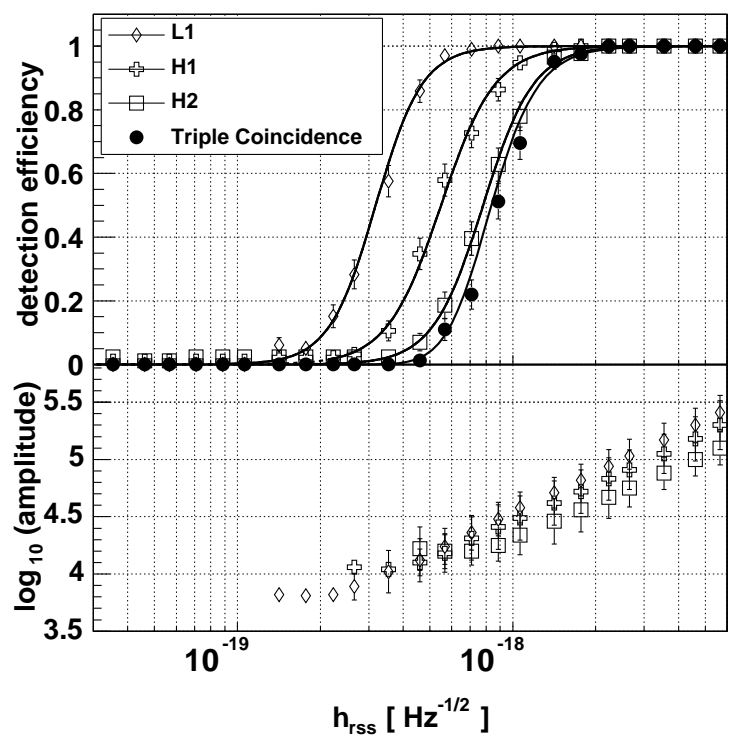

FIG. 11: This is the same as Fig. 10 but obtained with the SLOPE event trigger generator.

$\mathrm{Hz}$ band in $\mathrm{L} 1$, where, for example, a $361 \mathrm{~Hz}$ sine-Gaussian with $h_{r s s} \gtrsim 6 \times 10^{-18}$ could begin to trigger the BLRMS cut. Signals centered at other frequencies or those with broader bandwidths would require a higher amplitude. We estimate that a loud supernova [40,41] at $7 \mathrm{pc}$, or a $3+3$ solar mass binary neutron star inspiral at $300 \mathrm{pc}$ could begin to trigger the BLRMS cut. (Note however that the well-defined waveform of the latter makes a template-based search [12] a more sensitive method for detecting such waves.) The need for this cut in the data was driven by the nonstationarity of the noise in the detectors during S2, and detector improvements are expected to reduce our use of such cuts in the future.

In Fig. 1 we compare the value of $h_{r s s}$ for which our simulations of sine-Gaussian waveforms (at optimal wave direction and polarization) yield 50\% efficiency (averaged over the entire S1 run), shown as circles, with the detectors' (typical) equivalent strain noise. These $50 \%$ efficiency points are roughly an order of magnitude larger than the equivalent strain noise.

\section{Average over direction and polarization}

The response of a LIGO detector to an incident gravitational wave burst depends on the wave direction and wave polarization relative to the detector axes, and is referred to as the detector's antenna pattern [15]. The only effects of the wave direction and polarization are to modify the amplitude of the detected wave and the relative arrival times at the detectors. Since we have evaluated the detection efficiency for each detector as a function of the root-sum-square strain of the wave at optimal direction (directly overhead) and polarization (aligned with the detector axes), it is straightforward to evaluate the efficiency at arbitrary direction and polarization. We 
choose to consider a population of sources distributed isotropically in the sky, with random linear polarization. We thus evaluate the detection efficiency averaged over direction and polarization, as a function of intrinsic strain per root $\mathrm{Hz}$ incident on the Earth.

In order to evaluate the efficiency for coincident detection by non-colocated detectors, we assume that the detection efficiency is a measure of a random process, uncorrelated between detectors. Further, the difference in arrival times at the different detectors is small compared to the time coincidence window employed (section \IIF 1. Therefore, the efficiency for triple coincidence can be expressed as the product of efficiencies for the three LIGO detectors evaluated at the appropriate peak amplitude for each. Under this assumption, the efficiency for coincident detection by all three LIGO detectors, averaged over wave direction and polarization, can be evaluated numerically. The results of this procedure are shown in Figs. 12, 13 and 14

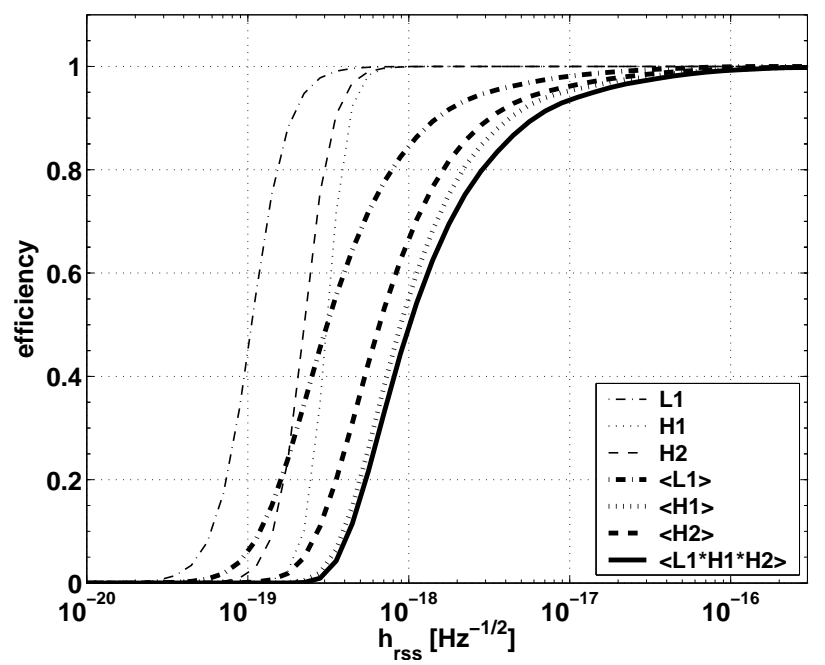

FIG. 12: Burst detection efficiency for Gaussian bursts with $\tau=1$ $\mathrm{ms}$, as a function of $h_{r s s}$, for each of the three LIGO detectors, and for the triple coincidence, using the TFCLUSTERS event trigger generator. The lighter grey curves are the same as the curves in Fig. 10 The darker curves to the right of them are the result of averaging the efficiency curves over wave directions and polarizations (denoted by $\langle\ldots\rangle$ in the legend) as described in section $\nabla \mathrm{VC}$

The single-detector efficiencies will be independent only if there are no significant noise correlations between the detectors. We have compared the results for coincident detection to direct simulations of triple coincidence through the full threedetector analysis pipeline (under the simplifying assumption of neglecting the difference in antenna pattern response between the two sites), and found good agreement (see triple coincidence data points in Figs. 10 and 11); no evidence for burst detection efficiency correlations between the detectors has been observed.

A crucial test of the accuracy of this simulation procedure is the comparison of signals injected into the data stream with software, with signals injected directly into the end test masses of the interferometers. The comparison of these "hard-

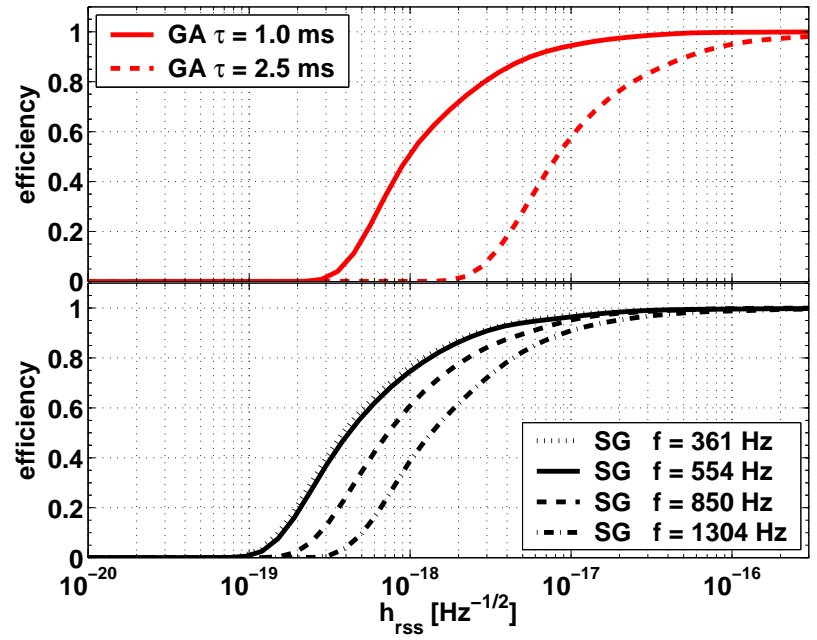

FIG. 13: Burst detection efficiency for triple coincidence as a function of $h_{r s s}$, using the TFCLUSTERS event trigger generator, averaging over wave directions and polarizations, for six different waveforms: GA refers to the Gaussians defined in Eqn. 5.1 and SG to the sine-Gaussians defined in Eqn. 5.2

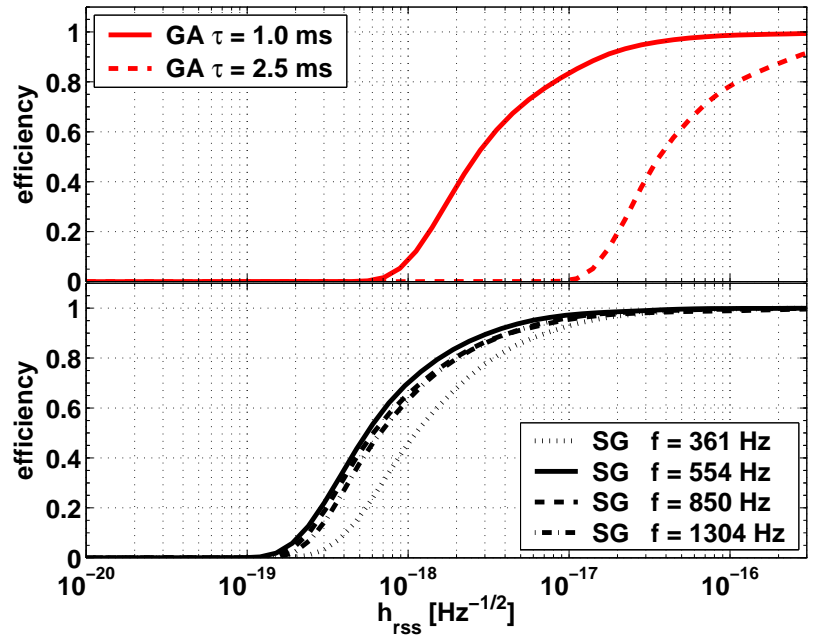

FIG. 14: Same as Fig.13 for the SLOPE event trigger generator.

ware" burst injections with the (much more numerous) software injections provide a test of the detector response, the calibration information, the data acquisition, and the entire software analysis chain, including the software simulations used to evaluate the efficiency, as described above.

Hardware injections of sine-Gaussians with varying $f_{0}$ and $h_{r s s}$ were performed during brief periods, just prior to the beginning and just after the end of the S1 run. Due to time constraints, only a limited number of hardware injections were performed. As a result, the comparison with software injections is somewhat qualitative. The detection of these hardware injections through the analysis pipeline was found to be consistent with expectations from the software injections. 


\section{Sensitivity to modeled bursts}

We can use the efficiency determined by simulated signal injections, discussed in sections $\mathrm{VB}$ and $\mathrm{VC}$ to estimate the weakest signal we could have seen in the search described in this article. The efficiencies of each of our two event trigger generators for several different waveforms is shown in Figure 13 and Figure 14. The sensitivity at 50\% efficiency, for a variety of Gaussian and sine-Gaussian waveforms, is shown in Table in terms of $h_{r s s}$, and in Table in terms of $h_{\text {char }}$.

TABLE II: Sensitivity to various waveforms in the S1 run from TFCLUSTERS and SLOPE pipelines for triple-coincidence detection, averaged over source direction and polarization. The sensitivity is given in terms of $h_{r s s}$ (Eqn.5.3 units of $\mathrm{Hz}^{-1 / 2}$ ), at $50 \%$ efficiency $\left(h_{r s s 1 / 2}\right)$. A $20 \%$ uncertainty associated with calibration (section VIC is not included.

\begin{tabular}{lcc}
\hline \hline & $\begin{array}{c}\text { TFCLUSTERS } \\
{\left[\mathrm{Hz}^{-1 / 2}\right]}\end{array}$ & $\begin{array}{c}\text { SLOPE } \\
{\left[\mathrm{Hz}^{-1 / 2}\right]}\end{array}$ \\
\hline Gaussian $\tau=1.0 \mathrm{~ms}$ & $1.0 \times 10^{-18}$ & $2.6 \times 10^{-18}$ \\
Gaussian $\tau=2.5 \mathrm{~ms}$ & $8.2 \times 10^{-18}$ & $3.6 \times 10^{-17}$ \\
sine-Gaussian $f_{0}=153 \mathrm{~Hz}$ & $1.6 \times 10^{-18}$ & $1.2 \times 10^{-17}$ \\
sine-Gaussian $f_{0}=254 \mathrm{~Hz}$ & $5.1 \times 10^{-19}$ & $2.8 \times 10^{-18}$ \\
sine-Gaussian $f_{0}=361 \mathrm{~Hz}$ & $3.8 \times 10^{-19}$ & $1.1 \times 10^{-18}$ \\
sine-Gaussian $f_{0}=554 \mathrm{~Hz}$ & $4.2 \times 10^{-19}$ & $5.6 \times 10^{-19}$ \\
sine-Gaussian $f_{0}=850 \mathrm{~Hz}$ & $7.3 \times 10^{-19}$ & $6.1 \times 10^{-19}$ \\
sine-Gaussian $f_{0}=1304 \mathrm{~Hz}$ & $1.4 \times 10^{-18}$ & $6.7 \times 10^{-19}$ \\
sine-Gaussian $f_{0}=2000 \mathrm{~Hz}$ & $2.3 \times 10^{-18}$ & $2.5 \times 10^{-18}$ \\
\hline \hline
\end{tabular}

TABLE III: Sensitivity to various waveforms in the S1 run from TFCLUSTERS and SLOPE pipelines for triple-coincidence detection, averaged over source direction and polarization. The sensitivity is given in terms of $h_{\text {char }}$ (Eqn. 5.6 dimensionless strain), at 50\% efficiency. A $20 \%$ uncertainty associated with calibration (section VIC is not included.

\begin{tabular}{lcc}
\hline \hline & TFCLUSTERS & SLOPE \\
\hline Gaussian $\tau=1.0 \mathrm{~ms}$ & $1.4 \times 10^{-18}$ & $3.6 \times 10^{-18}$ \\
Gaussian $\tau=2.5 \mathrm{~ms}$ & $3.3 \times 10^{-19}$ & $1.5 \times 10^{-18}$ \\
sine-Gaussian $f_{0}=153 \mathrm{~Hz}$ & $3.1 \times 10^{-17}$ & $2.4 \times 10^{-16}$ \\
sine-Gaussian $f_{0}=254 \mathrm{~Hz}$ & $1.2 \times 10^{-17}$ & $6.8 \times 10^{-17}$ \\
sine-Gaussian $f_{0}=361 \mathrm{~Hz}$ & $1.1 \times 10^{-17}$ & $3.3 \times 10^{-17}$ \\
sine-Gaussian $f_{0}=554 \mathrm{~Hz}$ & $1.6 \times 10^{-17}$ & $2.1 \times 10^{-17}$ \\
sine-Gaussian $f_{0}=850 \mathrm{~Hz}$ & $3.4 \times 10^{-17}$ & $2.8 \times 10^{-17}$ \\
sine-Gaussian $f_{0}=1304 \mathrm{~Hz}$ & $8.0 \times 10^{-17}$ & $3.8 \times 10^{-17}$ \\
sine-Gaussian $f_{0}=2000 \mathrm{~Hz}$ & $1.6 \times 10^{-16}$ & $1.8 \times 10^{-16}$ \\
\hline \hline
\end{tabular}

\section{INTERPRETED RESULTS}

\section{A. Exclusion in rate versus strength plane}

The results of our search can be used to set limits on models of ensembles of gravitational waves arriving at the earth. Fig. 15 shows the upper limits that we set, using the TFCLUSTERS event trigger generator, as expressed in the plane of event rate versus $h_{r s s}$. The top figure is for the case of $1 \mathrm{~ms}$ and $2.5 \mathrm{~ms}$ Gaussian bursts, and the lower figure is for sineGaussian bursts with central frequency of 361, 554, 850 and $1304 \mathrm{~Hz}$.

As discussed in sections $\llbracket$ and $\nabla$ these limits are given in terms of an ensemble of waves of equal amplitude, incident on the earth from all directions and with all (linear) polarizations. This ensemble is not motivated by astrophysical considerations, but is nevertheless useful in characterizing the performance of the search, and it can be compared with similar limits obtained by resonant bar detector collaborations [1, 2].

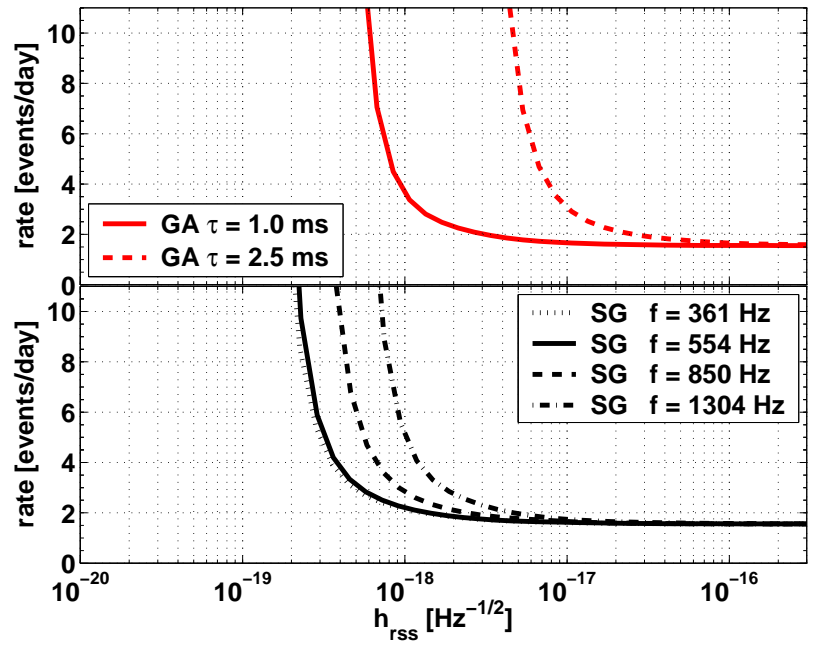

FIG. 15: Rate versus $h_{r s s}$ for detection of specific waveforms using the TFCLUSTERS event trigger generator. The region above and to the right of the curves is excluded at $90 \%$ confidence level or greater. The effect of the $20 \%$ uncertainty in the detector response is included. Top: For Gaussians with $\tau=1.0 \mathrm{~ms}$ and $\tau=2.5 \mathrm{~ms}$. Bottom: For sine-Gaussians with $Q=9$ and central frequency $f_{0}=$ 361, 554, 850 and $1304 \mathrm{~Hz}$.

The curves in Fig. 15 are constructed by dividing our observed event rate upper limit by the efficiency curves shown in Fig. 13 In the limit of large wave amplitudes $h_{r s s}$ where our efficiency is essentially unity, the upper limit is independent of amplitude, at a level given by the burst rate limit presented in sectionIVC The limit at all amplitudes with lower efficiency is given by that burst rate limit, multiplied by the inverse of the efficiency.

The meaning of the upper limit curve can be understood by imagining that one is interested in the flux of $1 \mathrm{~ms}$ Gaussian gravitational wave bursts at a particular amplitude. Reading the value of the curve at that amplitude gives the $90 \%$ confidence level upper limit on the rate of such bursts with $h_{r s s}$ 
equal to or greater than that amplitude. Similarly, one can use these curves to determine the $90 \%$ confidence level upper limit on the amplitude of bursts which are incident at a specified rate.

\section{B. Comparing results from the two pipelines}

As discussed in section IVA the variability in the SLOPE background led us to choose not to use it to set an upper limit on the gravitational wave burst rate. However, the two event trigger generators use very different and complementary methods to identify bursts in the data, and it is thus interesting to compare the results from the two pipelines. We want to make the strongest statement that we can about the rate of gravitational wave bursts, consistent with sound statistical practice. We evaluated the efficiency of our two event trigger generator pipelines for each of our candidate signal waveforms, tuned to give approximately the same background rate using the playground data set. To combine the results from the two pipelines, we would choose to quote upper limits from the event trigger generator that has the better efficiency for the largest number of waveforms. With the tuning parameters used in this study, we find that the TFCLUSTERS pipeline has better efficiency than SLOPE for most of these waveforms (Table III), although SLOPE performs somewhat better for the $850 \mathrm{~Hz}$ and $1304 \mathrm{~Hz}$ sine-Gaussians. Thus independent of the decision to not use the SLOPE result because of the problems with background variability, the final upper limits that we would set are the ones obtained from the TFCLUSTERS pipeline, shown in Fig. 15] The SLOPE results provide a valuable cross-check, and we intend to continue to employ and improve the SLOPE pipeline in future analyses (section VIIB).

\section{Systematic uncertainties}

The following systematic uncertainties are known to significantly contribute to systematic errors associated with the results of our search. The estimation of our efficiency for detection of bursts with modeled waveforms (the Gaussians and sine-Gaussians that were considered in section $\mathrm{VB}$ has a statistical error associated with the finite number of simulations. The underlying noise floor was modeled using a sample of the $\mathrm{S} 1$ data itself; there is a systematic uncertainty associated with the degree to which this sample was representative of the entire S1 dataset.

The analysis procedure for the efficiency has various potential sources of error. The detection efficiency versus $h_{r s s}$ is fitted with a sigmoid curve, as discussed in section $\mathrm{VB}$ The efficiency curves for each detector are combined to get an overall triple-coincidence efficiency, averaging over source direction and polarization, assuming that the coincident efficiency is the product of the individual efficiencies (as a function of amplitudes at each detector). The effects of our (very limited) post-coincidence processing, including the choice of coincident time windows, clustering of multiple triggers from a single detector, and consistency of burst trigger parameters from the three detectors, have been studied. None of these studies reveal a significant source of error. It should be noted here that future results will employ far more detailed postcoincidence processing (see section VIIB, including much tighter coincident time windows, and these issues will be carefully re-evaluated.

By far the largest source of systematic error associated with the efficiency determination is the uncertainty in the detector response to gravitational waves as obtained through the calibration procedure [27, 28]. We have combined the estimated uncertainty in the DC calibration with potential uncertainties in the frequency response, convolved with the frequency spectra of our modeled waveforms. We conservatively estimate an overall systematic uncertainty of $20 \%$ in the strain scale for our efficiency curves. Our curves of upper-limit versus gravitational wave strain (Fig. 15) reflect this uncertainty by assuming that the detectors' response is $20 \%$ less sensitive than the nominal value.

We assign no systematic error associated with our choice of modeled waveforms since these are used to define the set of bursts which are targeted by this search.

We assign no systematic errors associated with our choice of data used or our BLRMS cut or calibration cut (section IIC. These procedures simply fix our observation time.

The upper limit on the number of observed bursts depends on our estimate of the backgrounds, as discussed in section IV The statistical errors associated with these estimations are marginalized over in the process of establishing the confidence bands; since these errors are small, this has a negligible effect on the resulting upper limits. We have performed a variety of studies to search for and estimate sources of systematic errors in the background estimate, including the time dependence of the background rate, various time-lag procedures, excluding anomalous data stretches, and other consistency checks. No sources of additional systematic error associated with the background rate are found to be significant.

\section{SUMMARY AND DISCUSSION}

We have searched for gravitational wave bursts of astrophysical origin using data taken during the first Science Run of the three LIGO detectors. A total of 35.5 hours of triple coincidence observation time satisfied our data quality requirements.

We employed two different algorithms for the identification of candidate burst events in the gravitational wave data channel. With the algorithm for which we chose to present a final result (the TFCLUSTERS event trigger generator), we observe numbers of events that are reasonably consistent with expectations for random accidental coincidences of events originating from noise fluctuations in the three detectors. We thus limit the excess event rate to be less than 1.6 per day, at $90 \%$ confidence level.

We estimate our efficiency for the detection of linearly polarized gravitational wave bursts incident on the detector array with a range of amplitudes and averaged over source di- 
rection and wave polarization, by injecting simulated signals into the raw S1 data streams and performing the search as it is done on the raw data itself. We focus on two simple, ad hoc waveform morphologies (section $\nabla \mathrm{A}$ ): Gaussians with a range of durations, and sine-Gaussians with a $\mathrm{Q}$ of 9 , and a range of central frequencies. With these, we evaluate the (waveform-dependent) detection efficiencies as a function of strain per root hertz, $h_{r s s}$. We then combine the rate limit with the efficiencies to establish exclusion regions in the rateversus-strength plane; representative examples are shown in Fig. 15 These constitute the results of the search reported in this paper.

\section{A. Comparison with other burst searches}

Searches for gravitational wave bursts have been a main focus of the observational program of the resonant-mass detector community, and our work was able to benefit from their prior work. The most recent analysis by the IGEC consortium [4], which includes data from five detectors spread around the world, has presented its upper limits for bursts in the form of a rate-amplitude diagram, in much the same style as our Fig.15

The IGEC result (Fig. 13 of [4]) bounds the rate for GW burst events with large amplitude to be less than $\sim 4 \times 10^{-3}$ events per day. This rate bound is much stronger than the ones reported here due to the much longer observation time of the IGEC run. The rate bound gets rapidly worse for lower amplitude bursts, due to increasing background and decreasing detection efficiency.

To crudely compare the sensitivity of the two searches to low-amplitude bursts, we can define a "detection threshold" as the value of the burst amplitude at which the rate limit is two times worse than the limit for large amplitude bursts. For the analysis presented here, this is the $50 \%$ efficiency point $h_{\text {rss } 1 / 2}$ reported in Table

One difference between the IGEC work and ours is that their instruments have relatively narrow frequency bandwidths, and so are sensitive to a different measure of burst strength. Their detectors measure the Fourier magnitude $\left|\tilde{h}\left(f_{b}\right)\right|$ of a signal waveform at the bars' resonant frequency $f_{b} \approx 900 \mathrm{~Hz}$, incident at optimal source direction and polarization.

However, if we consider a specific waveform with frequency content dominantly at or near at $f_{b}$, such as our sineGaussians with central frequency $f_{0}=850 \mathrm{~Hz}$, the bar sensitivities and the interferometric detectors' sensitivities can be directly compared, over the relatively narrow frequency band where bar detectors are most sensitive. The conversion from $\left|\tilde{h}\left(f_{b}\right)\right|$ to $h_{r s s}$ for bars for the sine-Gaussian family of signals (Eqn.5.2) is calculated to be

$$
h_{r s s}=\frac{\left|\tilde{h}\left(f_{b}\right)\right|}{\sqrt{\tau}} \frac{\left(\frac{2}{\pi}\right)^{\frac{1}{4}}}{\left(e^{-\pi^{2} \tau^{2}\left(f_{b}-f_{0}\right)^{2}}-e^{\left.-\pi^{2} \tau^{2}\left(f_{b}+f_{0}\right)^{2}\right)}\right.} .
$$

We focus on sine-Gaussians with central frequency $f_{0}=$ $850 \mathrm{~Hz}$, incident at optimal direction and polarization. Using Fig. 13 of [4] and Eqn. 7.1] the IGEC detection threshold is roughly $h_{r s s} \approx 1 \times 10^{-19} \mathrm{~Hz}^{-1 / 2}$. To compare this with our sensitivity for $850 \mathrm{~Hz}$ sine-Gaussian bursts $\left(h_{r s s 1 / 2}=\right.$ $7.3 \times 10^{-19} \mathrm{~Hz}^{-1 / 2}$, Table II), we must first correct for our averaging over direction and polarization (section $\mathrm{VC}$. This yields an amplitude at $50 \%$ efficiency for waves with optimal orientation of $h_{r s s} \approx 2.6 \times 10^{-19} \mathrm{~Hz}^{-1 / 2}$. Note that this detection threshold for LIGO is established by determining the loss of efficiency for fixed threshold, while for IGEC it is established by observing an increase in background events as the threshold is varied. Nonetheless, this measure of detection threshold permits a rough comparison of the search sensitivites [43], and we see that the IGEC search [4] has a somewhat greater sensitivity to $850 \mathrm{~Hz}$ sine-Gaussian bursts than the one presented here.

For all other waveforms shown in Fig. 15 and for other waveforms with significant spectral amplitude in a broad range of frequencies away from $f_{b}$, the LIGO constraints are more stringent than the IGEC results, due to the broad band response of the interferometric detectors. For sine gaussians at $554 \mathrm{~Hz}$, the ratio of peak spectral density of the pulse to the spectral density in the resonant-mass detector band is less than $10 \%$ for NIOBE and negligible in the other four IGEC detectors. For sine gaussians at $1304 \mathrm{~Hz}$, ALLEGRO, AURIGA, EXPLORER and NAUTILUS receive spectral densities in their bands that are only a few percent of peak spectral density for the pulse, with negligible spectral density in NIOBE's band. The resonant mass detectors also receive relatively small spectral density in their bands from gaussian waveforms compared to the LIGO detectors, unless $\tau$ is less than $1 \mathrm{~ms}$. This emphasises the importance of broad band sensitivity in searching for unmodeled gravitational-wave bursts. Ongoing work to broaden the response of resonant mass detectors should improve sensitivity to other waveforms in the future.

The only previously published results on searches for burst events with broadband interferometric detectors that we are aware of are in Ref. [5] (but see also Ref. [6]). In Ref. [5], prototype detectors developed by the University of Glasgow and Max Planck Institute for Quantum Optics were operated for an effective coincident observing period of 62 hours in 1989. They searched for bursts with significant frequency content in the band from 800 to $1250 \mathrm{~Hz}$. They considered the waveform $h(t)=h_{\text {peak }} \sin \left(2 \pi f_{m} t\right) /\left(2 \pi f_{m} t\right)$ [44], which has constant Fourier magnitude from 0 to $f_{m}=1250 \mathrm{~Hz}$, and in-band $(\Delta f=1250-800=450 \mathrm{~Hz})$ root-sum-square amplitude $h_{r s s}=\sqrt{2 \Delta f} /\left(2 f_{m}\right) h_{\text {peak }}$. They observe no events with $h>h_{\text {peak }}=4.9 \times 10^{-16}$, or $h_{\text {rss }}=5.9 \times 10^{-18} \mathrm{~Hz}^{-1 / 2}$, averaging over wave polarizations and incident directions. Therefore, they set an upper limit on the rate of bursts with strain greater than this value, of $0.94 /$ day. Their sensitivity can be compared with the strain sensitivities reported here at $50 \%$ efficiency, for sine-Gaussians with central frequencies of

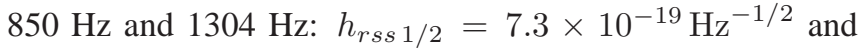
$h_{\text {rss } 1 / 2}=1.4 \times 10^{-18} \mathrm{~Hz}^{-1 / 2}$, respectively (Table $\amalg$. 


\section{B. Directions for improved analysis in the future}

LIGO's second science run (S2) accumulated data for 8 weeks in early 2003. At most frequencies, the noise in the three LIGO detectors was improved compared to the noise level of the S1 data presented here by a factor of 10 . Some improvements in the stability of the noise were also achieved. The in-lock duty cycles of the detectors were comparable to those obtained during S1, but tighter monitoring of the detectors' noise levels and calibration should lead to significantly less loss of data than was suffered in S1. Even without improvements in our analysis methodology, we expect to obtain results from the $\mathrm{S} 2$ data that are an order of magnitude more sensitive in amplitude, and observation times that are increased by at least a factor of four over the results presented here.

Based on lessons learned during the $\mathrm{S} 1$ analysis, we are preparing numerous improvements and additions to our search methodology for the S2 data set. The pipeline presented here can be improved with more attention to optimizing and characterizing our event trigger generators. Obvious areas for improvement are better prefiltering, and better time resolution for both SLOPE and TFCLUSTERS. We have also implemented an adaptive threshold for the SLOPE event trigger generator to make its event rate less sensitive to variations in detector noise. As the detector performance becomes more stable and closer to the design sensitivity, safe and effective vetoes based on auxiliary channels that monitor the environment and interferometer sensing and control will be applied to reduce the number of spurious event triggers. New event trigger generators, using a variety of detection techniques (e.g., that proposed in [45]), will be tested.

After initial (coarse) identification of coincident events with improved versions of the event trigger generators, the gravitational wave data channel time series can be reexamined to further reduce the background of accidental coincidences. Crosscorrelation of the gravitational wave channels from multiple detectors can tighten our coincidence window so that it is lim- ited only by the light-travel time between detector sites, and test whether the event amplitudes and waveforms are consistent with the common origin of a gravitational plane wave.

Future searches will include a more astrophysical style of interpretation, setting limits on populations of events in three-dimensional space. Efficiency simulations will include more realistic waveforms, such as black hole ringdowns or supernova waveforms [40, 41]. Higher sensitivity for modeled bursts can be obtained using matched filter techniques. Longer runs will give more opportunities to search for gravitational wave bursts coincident with gamma ray burst events, using the methodology described in [46].

Finally, and crucially, we are developing criteria by which we can establish confidence in the detection of gravitational wave bursts both statistically and as a single large amplitude burst event. For single burst event candidates, we will use information from all available detectors to reconstruct our best estimates of the gravitational wave direction, polarization, and waveform.

\section{Acknowledgments}

The authors gratefully acknowledge the support of the United States National Science Foundation for the construction and operation of the LIGO Laboratory and the Particle Physics and Astronomy Research Council of the United Kingdom, the Max-Planck-Society, and the State of Niedersachsen/Germany for support of the construction and operation of the GEO600 detector. The authors also gratefully acknowledge the support of the research by these agencies and by the Australian Research Council, the Natural Sciences and Engineering Research Council of Canada, the Council of Scientific and Industrial Research of India, the Department of Science and Technology of India, the Spanish Ministerio de Ciencia y Tecnologia, the John Simon Guggenheim Foundation, the David and Lucile Packard Foundation, the Research Corporation, and the Alfred P. Sloan Foundation. This document has been assigned LIGO Laboratory document number LIGO-P030011.
[1] Z. Allen et al., Phys. Rev. Lett. 85, 5046 (2000).

[2] P. Astone et al., Class. Quant. Grav. 19, 5449 (2002).

[3] IGEC, P. Astone, et al., Class. Quant. Grav. 19, 1367 (2002).

[4] International Gravitational Event Collaboration (IGEC), Phys. Rev. D 68, 022001 (2003).

[5] D. Nicholson et al., Phys. Lett. A218, 175 (1996).

[6] R. Forward, Phys. Rev. D17, 379 (1978).

[7] A. Abramovici et al., Science 256, 325 (1992).

[8] GEO600, B. Willke, et al., Class. Quant. Grav. 19(7), 1377 (2002).

[9] GEO600, S. Goßler, et al., Class. Quant. Grav. 19(7), 1835 (2002).

[10] TAMA, H. Tagoshi, et al., Phys. Rev. D 63, 062001 (2001).

[11] VIRGO, F. Acernese, et al., Class. Quant. Grav. 19, 1421 (2002).

[12] LIGO Scientific Collaboration, Analysis of LIGO data for gravitational waves from binary neutron stars, gr-qc/0308069, sub- mitted to Phys. Rev. D (2003).

[13] LIGO Scientific Collaboration, Setting upper limits on the strength of periodic gravitational waves using the first science data from the GEO600 and LIGO detectors, gr-qc/0308050, submitted to Phys. Rev. D (2003).

[14] LIGO Scientific Collaboration, Analysis of First LIGO Science Data for Stochastic Gravitational Waves, To be submitted to Phys. Rev. D (2003).

[15] K. S. Thorne, in 300 Years of Gravitation, edited by S. Hawking and W. Israel (Cambridge University Press, Cambridge, 1987), chap. 9, pp. 330-458.

[16] P. R. Saulson, Fundamentals of Interferometric Gravitational Wave Detectors (World Scientific Pub. Co., 1994).

[17] LIGO Scientific Collaboration, Detector Description and Performance for the First Coincidence Observations between LIGO and GEO, gr-qc/0308043, accepted by Nucl. Instr. Meth. A (2003). 
[18] R. Savage, P. King, and S. Seel, Laser Physics 8, 679 (1998).

[19] S. Kawamura, A. Abramovici, and M. Zucker, Rev. Sci. Instr. 68, 223 (1997).

[20] S. Whitcomb et al., Optics development for LIGO (1997), in Proceedings of the TAMA International Workshop on Gravitational Wave Detection, November 11-12, 1996, Saitama, Japan, K. Tsubono, M.-K. Fujimoto, and K. Kuroda, eds., Universal Academy Press, Inc., Tokyo.

[21] C. Walsh et al., Appl. Optics 38, 2870 (1999).

[22] M. Barton, J. Romie, and S. Kawamura (1997), LIGO Technical Reports T970158-06-D and T970135-02-D, http://www.ligo.caltech.edu/docs/T/T970158-06.pdf and http://www.ligo.caltech.edu/docs/T/T970135-02.pdf.

[23] J. Giaime, P. Saha, D. Shoemaker, and L. Sievers, Review of Scientific Instruments 67, 208 (1996).

[24] P. Fritschel, R. Bork, G. González, N. Mavalvala, D. Ouimette, H. Rong, D. Sigg, and M. Zucker, Applied Optics 40, 4988 (2001).

[25] P. Fritschel, G. González, N. Mavalvala, D. Shoemaker, D. Sigg, and M. Zucker, Applied Optics 37, 6734 (1998).

[26] J. Giaime, E. Daw, M. Weitz, et al., Review of Scientific Instruments 74, 218 (2003).

[27] R. Adhikari et al., to be submitted to Class. Quant. Grav. (2003).

[28] R. Adhikari et al. (2003), LIGO Technical Report T030097-00D, http://www.ligo.caltech.edu/docs/T/T030097-00.pdf.

[29] This is an instance where our parameter tuning, event property estimation, or other analysis procedure was inadequately optimized prior to being frozen for the analysis of the full S1 data set. See the discussions at the beginning of section 111 and in section VIIB

[30] N. Arnaud, F. Cavalier, M. Davier, and P. Hello, Phys. Rev.
D59, 082002 (1999).

[31] T. Pradier, N. Arnaud, M. Bizouard, F. Cavalier, M. Davier, and P. Hello, Phys. Rev. D 63, 42002 (2001).

[32] J. Sylvestre, Ph.D. thesis, Massachusetts Institute of Technology, Cambridge, Massachusetts (2002).

[33] J. Sylvestre, Phys. Rev. D 66, 102004 (2002).

[34] LIGO Data Analysis System (LDAS) version 0.4.0, http://www.ldas-cit.ligo.caltech.edu; LIGO Algorithm Library LAL/LALWrapper version post-S1-2002-09-24, http://www.lsc-group.phys.uwm.edu/lal/.

[35] S. Rice, Bell System Technological Journal 23, 282 (1944).

[36] LIGO Hanford Observatory, http://www.ligo-wa.caltech.edu/.

[37] G. J. Feldman and R. D. Cousins, Phys. Rev. D 57, 3873 (1998).

[38] J. Conrad, O. Botner, A. Hallgren, and C. P. de los Heros, Phys. Rev. D 67, 012002 (2003).

[39] J. Conrad, O. Botner, A. Hallgren, and C. P. de los Heros, Coverage of confidence intervals for poisson statistics in presence of systematic uncertainties, hep-ex/0206034 (2002), to appear in Proceedings of Conference on Advanced Statistical Techniques in Particle Physics, Durham 2002.

[40] T. Zwerger and E. Müller, Astron. Astrophys. p. 209 (1997).

[41] H. Dimmelmeier, J. Font, and E. Müller, Astrophys. J. Lett. p. L163 (2001).

[42] E. Flanagan and S. Hughes, Phys. Rev. D57, 4539 (1998).

[43] W. Johnson and L. Baggio (IGEC), private communications.

[44] Bernard Schutz, private communication, July 18, 2003.

[45] W. Anderson, P. Brady, J. Creighton, and E. Flanagan, Phys. Rev. D 63, 042003 (2001).

[46] L. Finn, S. Mohanty, and J. Romano, Phys. Rev. D 60, 121101 (1999). 